\title{
LINK HOMOTOPY WITH ONE CODIMENSION TWO COMPONENT
}

\author{
PAUL A. KIRK
}

\begin{abstract}
Link maps with one codimension two component are studied and an invariant of link maps modulo link homotopy is constructed using ideas from knot theory and immersion theory. This invariant is used to give examples of nontrivial link homotopy classes and to show that there are infinitely many distinct link homotopy classes in many dimensions. A link map with the codimension two component embedded is shown to be nullhomotopic. These ideas are applied to the special case of 2-spheres in $S^{4}$ to give simple examples of the failure of the Whitney trick in dimension 4 .
\end{abstract}

Consider the following linking problem: Given two maps $f$ and $g$ from a union of manifolds $M_{1} \cup M_{2}$ into another manifold $N$ with $f\left(M_{1}\right) \cap f\left(M_{2}\right)=$ $\varnothing=g\left(M_{1}\right) \cap g\left(M_{2}\right)$, when does there exist a homotopy $F_{t}$ of $f$ to $g$ such that $F_{t}\left(M_{1}\right) \cap F_{t}\left(M_{2}\right)=\varnothing$ for all $t \in[0,1]$ ? Put another way, what is the structure of the set

$$
L M\left(M_{1}, M_{2} ; N\right)=\pi_{0}\left\{f: M_{1} \cup M_{2} \rightarrow N \mid f\left(M_{1}\right) \cap f\left(M_{2}\right)=\varnothing\right\} ?
$$

Link homotopy theory is the study of this problem. Map $f: M_{1} \cup M_{2} \rightarrow N$ with $f\left(M_{1}\right) \cap f\left(M_{2}\right)=\varnothing$ are called (two component) link maps. One can of course consider link maps with more than two components.

There are many variations of this problem, depending on the choices of the manifolds $M_{i}$ and $N$. It is natural to first restrict the manifold $N$ to be a sphere or Euclidean space, so that the homotopy properties of $N$ do not obscure the linking phenomena of the $M_{i}$. Another natural restriction is to assume that the dimension of the $M_{i}$ is less than the dimension of $N$, this avoids entering the realm of difficult homotopy theory problems (note that $L M\left(\mathbf{S}^{p}, \mathbf{S}^{m}\right)=$ $\pi_{p} \mathbf{S}^{m}$ ). This restriction makes the questions accessible to geometric methods.

An interesting special case occurs when the $M_{i}$ are spheres; in this case we write $L M_{p, q}^{m}$ for $L M\left(\mathbf{S}^{p}, \mathbf{S}^{q} ; \mathbf{S}^{m}\right)$. Since $L M_{p, q}^{m}$ "acts" on $L M\left(M_{1}, M_{2} ; N\right)$ by taking connected sums, it is clear that a good understanding of $L M_{p, q}^{m}$ is

Received by the editors August 25, 1988.

1980 Mathematics Subject Classification (1985 Revision). Primary 57R42.

Key words and phrases. Links, link homotopy, Whitney trick. 
needed before one can say anything about the general case. We will restrict attention to spherical link maps in this paper.

Milnor introduced this subject in 1954 in his paper Link groups [Mil]. He considered the link homotopy problem for circles in $\mathbf{S}^{3}$ and was able to give a criterion to decide when a link is homotopically trivial, that is, link homotopic to a map which sends each component to a different point in $\mathbf{S}^{3}$. In fact, he showed that if a certain quotient of the fundamental group of the link is isomorphic to the corresponding quotient of the trivial link, then the link is homotopically trivial.

There has been a renewed interest in this subject, particularly in the higherdimensional cases (see [Sc, Kol-Ko4, MR, FR]). The papers of Scott [Sc] and Massey and Rolfsen [MR] give a good description of higher dimensional link maps up to homotopy when the codimension of each component is greater than two. As usual, the fundamental group of the complement of codimension two objects complicates the situation and their techniques did not say much about the sets $L M_{p, m-2}^{m}$. A paper of R. Fenn and D. Rolfsen [FR] gave an example of a link map of two spheres in $\mathbf{S}^{4}$ which is not homotopically trivial; this was the only interesting example of a link map with one codimensional two component.

In this paper we develop methods to deal with link maps with codimension two components. Our approach to this problem is geometric and combines ideas from knot theory and immersion theory to define polynomial type invariants for link maps. These polynomials have recently found applications to embedded link theory $[\mathrm{J}]$ via a "suspension" construction which produces link maps from embedded links.

It has become clear that the study of link maps breaks down to roughly two questions:

1. When is a link map link homotopic to a link map which embeds one or both components?

2. When is a link map with one or both components link-homotopically trivial?

In a previous paper [Ki2], we considered the second question and showed that embedded links $\mathbf{S}^{p} \cup \mathbf{S}^{m-2} \subset \mathbf{S}^{m}$ are nullhomotopic if $p \leq \frac{2}{3} m-4$. We will consider the first problem here. Our approach is to take a "generic" representative of a link map and to study its double point manifold. Viewed in an appropriate bordism group, this double point manifold provides an obstruction to changing the link map to an embedded link by a link homotopy.

The idea of studying link maps via their double point (and $r$-tuple point) sets has been fruitful. Koschorke [Ko4] has recently used bordism invariants of the multiple point sets to fit some of the $L M_{p, q}^{m}$ into exact sequences; he also has found a host of invariants and has exhibited intricate connections between them.

$\S 2$ contains the basic definitions, as well as a few technical results which allow us to consider only immersed link maps and regular link homotopies. In 
$\S 3$ the $\sigma$ polynomial is introduced, and several of its properties are discussed. In particular we show that $\sigma$ is additive with respect to connected sums of links, a fact we can exploit to show that $L M_{p, 2 p-2}^{2 p}$ is a group and to show that $L M_{p, m-2}^{m}$ is infinite for many values of $p, m$.

In $\S 4$ a general method for constructing link maps with $\sigma$ nonzero is described. This method is applied to show that $\sigma$ maps $L M_{p, 2 p-2}^{2 p}$ and $L M_{p, 2 p-3}^{2 p}$ onto infinitely generated groups. In $\S 5$ we prove a surprising theorem that if $f: \mathbf{S}^{p} \cup \mathbf{S}^{m-2} \rightarrow \mathbf{S}^{m}$ is a link map which embeds $\mathbf{S}^{m-2}$ and if $3 p \leq 2 m-4$, then $f$ is link homotopically trivial. In particular, $\sigma=0$. This implies that the double points of a $p$-sphere in a knot complement cannot link the knot. Alternatively, one can conclude that for certain maps $f: \mathbf{S}^{p} \rightarrow \mathbf{S}^{m}$, many homotopy classes in $\pi_{m-2}\left(\mathbf{S}^{m}-f\left(\mathbf{S}^{p}\right)\right)$ cannot be represented by embedded spheres. In the course of the proof we define a "homotopy intersection pairing" $\pi_{p} X \times \pi_{q} X \rightarrow G\left[t, t^{-1}\right]$ for $X$ a knot complement. This generalizes the usual homological intersection pairing in the infinite cyclic cover of a knot.

In $\S 6$ we consider the special case of two-spheres in $\mathbf{S}^{4}$. It is conjectured that a link map $\mathbf{S}^{2} \cup \mathbf{S}^{2} \rightarrow \mathbf{S}^{4}$ which embeds the first component is nullhomotopic. We show that $\sigma$ vanishes if just one of the spheres is embedded, giving evidence for this conjecture. This result is used to give many simple examples of the failure of the Whitney trick in dimension 4 . We conclude with some comments on multiple component link maps and some questions.

I wish to thank J. Levine for his encouragement and helpful discussions while this work was in progress and also U. Koschorke for sending me preliminary versions of his work which inspired many of the ideas contained in this paper.

By a (spherical) link map we mean a map $f: \mathbf{S}^{p} \cup \mathbf{S}^{q} \rightarrow \mathbf{S}^{m}$ such that $f\left(\mathbf{S}^{p}\right) \cap$ $f\left(\mathbf{S}^{q}\right)=\varnothing$. One can also consider more general manifolds, but we will devote most of our attention to spherical link maps. Two link maps are called link homotopic if they are homotopic through link maps. In particular, we call a link map nullhomotopic if it is homotopic to the map sending $\mathbf{S}^{p}$ to the north pole and $\mathbf{S}^{q}$ to the south pole of $\mathbf{S}^{m}$. Denote by $L M_{p, q}^{m}$ the set of link maps modulo link homotopy.

A useful invariant used to study $L M_{p, q}^{m}$ is the $\alpha$ invariant [Sc, MR] which can be defined as follows: given a link map $f: \mathbf{S}^{p} \cup \mathbf{S}^{q} \rightarrow \mathbf{S}^{m}$, pick a point $\infty \in \mathbf{S}^{m}-f\left(\mathbf{S}^{p} \cup \mathbf{S}^{q}\right)$ and identify $\mathbf{S}^{m}-\infty$ with $\mathbf{R}$. The map $\boldsymbol{\Phi}: \mathbf{S}^{p} \times \mathbf{S}^{q} \rightarrow \mathbf{S}^{m-1}$ given by

$$
\Phi(x, y) \mapsto \frac{f(x)-f(y)}{\|f(x)-f(y)\|}
$$

defines via the Hopf construction a map $\alpha: \mathbf{S}^{p} * \mathbf{S}^{q} \rightarrow \mathbf{S}^{m}$ whose homotopy class in $\pi_{p+q+1}\left(\mathbf{S}^{m}\right)$ is an invariant of the link homotopy class of $f$. If $p, q \leq m-2$, then $\pi_{p+q+1}\left(\mathbf{S}^{m}\right)$ is stable, and so in this case we consider $\alpha$ as an element of $\pi_{p+q+1-m}^{s}$. 
The following theorems are proven in [MR]; the first is due to J. Levine and the second to Kervaire.

Theorem 2.1. Suppose $1<p \leq m-2$ and $f \in L M_{p, m-2}^{m}$ is link homotopic to a map which embeds the codimension two component. Then $\alpha(f)=0$.

Theorem 2.2. Suppose $p<m-2$ and $f: \mathbf{S}^{p} \cup \mathbf{S}^{q} \rightarrow \mathbf{S}^{m}$ embeds $\mathbf{S}^{p}$. Note that $\mathbf{S}^{m}-f\left(\mathbf{S}^{p}\right)$ is homotopy equivalent to $\mathbf{S}^{m-p-1}$, so that $f: \mathbf{S}^{q} \rightarrow \mathbf{S}^{m}-f\left(\mathbf{S}^{p}\right)$ determines a class $\lambda \in \pi_{q}\left(\mathbf{S}^{m-p-1}\right)$. Then $\alpha=\sum^{\infty} \lambda$.

The class $\lambda$ is called the linking coefficient of the link. It is defined for any link map which embeds a component of codimension greater than two.

The invariant $\alpha$ is a sensitive invariant in studying the collection of embedded links modulo link homotopy. In particular the main result of [MR] states that $\alpha$ determines the link homotopy class of an embedded link if $p+q \leq \frac{3}{2} m-3$ and $p, q \leq m-3$. This was extended in [Ki2]: any embedded link $f: \mathbf{S}^{p} \cup$ $\mathbf{S}^{m-2} \rightarrow \mathbf{S}^{m}$ with $3 \leq \frac{3}{2} p \leq m-2$ is nullhomotopic. As a consequence of this theorem and Theorem 2.1 we see that $\alpha$ determines the link homotopy class of an embedded link with one codimension two component and one metastable component.

The situation for link maps is drastically different. In $\S 4$ we will show that $L M_{p, m-2}^{m}$ is an infinite set for many values of $p$ and $m$, so that $\alpha$ is far too weak an invariant to study the general type of link map.

We now discuss a few technical results which will allow us to reduce our study to link homotopies which are regular. The motivation for this is that under a regular link homotopy, the double point sets change by bordisms, and the invariants we later define will be bordism invariants of the double point manifolds. We remark here that for the rest of this paper, all link components will have codimension at least 2 .

From Hirsch's immersion theory [Hi] we know that any continuous function $f: \mathbf{S}^{p} \rightarrow \mathbf{S}^{m}$ can be approximated by an immersion, since spheres have trivial stable tangent bundles. (We will call $g$ an approximation of $f$ if $g$ is homotopic to $f$ in some small open neighborhood of $f\left(\mathbf{S}^{p}\right)$.) Thus we can always find a link homotopy from $f: \mathbf{S}^{p} \cup \mathbf{S}^{q} \rightarrow \mathbf{S}^{m}$ to a link map which immerses each component by perturbing each component slightly. This does not quite suffice for our needs. We wish to further perturb the link map so that the restriction of $f$ to each component is regularly homotopic to the standard embedding of a sphere in $\mathbf{S}^{m}$.

Lemma 2.3. 1. If $f: \mathbf{S}^{p} \rightarrow \mathbf{S}^{m}$ is an immersion, then $f$ can be approximated by an immersion $g$ which is regularly homotopic to the standard embedding $\mathbf{S}^{p} \subset \mathbf{S}^{m}$.

2. Let $U \subset \mathbf{S}^{m}$ be an open set. If $f_{0}, f_{1}: \mathbf{S}^{p} \rightarrow U$ are immersions which are homotopic in $U$ and regularly homotopic in $\mathbf{S}^{m}$, then they are regularly homotopic in $U$. 
Both statements follow from the consequence of Hirsch's theorem that there is exactly one regular homotopy class of immersions $g: \mathbf{S}^{p} \rightarrow U$ homotopic to $f$ and regularly homotopic to the standard embedding of $\mathbf{S}^{p}$ in $\mathbf{S}^{m}$.

We can now prove a theorem which enables us to reduce the study of link homotopy to that of regular link homotopy, at least in the case when the components are spheres.

Theorem 2.4. If $f_{0}, f_{1}: \mathbf{S}^{p} \cup \mathbf{S}^{q} \rightarrow \mathbf{S}^{m}$ are link homotopic maps such that the restrictions to each component are regularly homotopic to the standard embedding in $\mathbf{S}^{m}$, then there exists a regular link homotopy $f_{t}$ of $f_{0}$ to $f_{1}$. More generally a link homotopy between two link maps $f, g: \mathbf{S}^{p} \cup M^{m-2} \rightarrow \mathbf{S}^{m}$ where $M$ is not necessarily spherical and the restrictions to $\mathbf{S}^{p}$ are regularly homotopic to the standard embedding may be replaced by a link homotopy which is regular along $\mathbf{S}^{p}$.

Proof. We prove the first statement only, the proof of the second is easier. Using the compactness of $\mathbf{I}$ and the fact that any map $\mathbf{S}^{d} \rightarrow \mathbf{S}^{m}$ can be approximated by an immersion, it is easy to modify a link homotopy to obtain one satisfying:

- There exist numbers $0=i_{1}<\cdots<i_{k}=1$ so that $f_{t}$ is the stationary homotopy on $\mathbf{S}^{p}$ when $t \in\left[i_{2 l}, i_{2 l+1}\right]$ and the stationary homotopy on $\mathbf{S}^{q}$ when $t \in\left[i_{2 l-1}, i_{2 l}\right]$.

- The maps $f_{t}: \mathbf{S}^{p} \rightarrow \mathbf{S}^{m}, t \in\left[i_{2 l}, i_{2 l+1}\right]$ are regularly homotopic to the standard embedding, and similarly, $f_{t}: \mathbf{S}^{q} \rightarrow \mathbf{S}^{m}, t \in\left[i_{2 l-1}, i_{2 l}\right]$.

Using the second part of Lemma 2.3 , we can modify $f_{t}$ for $t \in\left[0, i_{1}\right]$ so that we replace the homotopy of $\mathbf{S}^{q}$ in $\mathbf{S}^{m}-f_{0}\left(\mathbf{S}^{p}\right)$ by a regular homotopy. Next we replace the homotopy of $\mathbf{S}^{p}$ in $\mathbf{S}^{m}-f_{i_{1}}$ by a regular homotopy. Continuing in this fashion we end up with a regular homotopy of $f_{0}$ to $f_{1}$ satisfying the conclusion of the theorem

Both Lemma 2.3 and Theorem 2.4 extend to the case of link maps with nonspherical components provided the manifolds have trivial stable tangent bundles and embed in $\mathbf{S}^{m}$.

We end this section with a lemma of Koschorke (Proposition 2.3 in [Ko2]). It will be used to show that $L M_{p, m-2}^{m}$ is often a group.

Lemma 2.5. If $p \leq m-2$, then $L M_{p, m-2}^{m}$ is a semigroup under connected sum.

We remind the reader that if $f, g: \mathbf{S}^{p} \cup \mathbf{S}^{m-2} \rightarrow \mathbf{S}^{m}$ are link maps, then $f \# g$ is the link map obtained by first placing $f$ and $g$ in different hemispheres of $\mathbf{S}^{m}$ by an isotopy, then choosing disjoint paths from $f\left(\mathbf{S}^{p}\right)$ to $g\left(\mathbf{S}^{p}\right)$ and $f\left(\mathbf{S}^{m-2}\right)$ to $q\left(\mathbf{S}^{m-2}\right)$, and finally forming a connected sum along thin, embedded tubes which follow these paths. Of course this procedure is not well defined, 
but Koschorke's lemma implies that the resulting link homotopy class is well defined.

In this section we will define our link homotopy invariant. To motivate the definition of the invariant, consider an immersed $\mathbf{S}^{p}$ in $\mathbf{S}^{m}-f\left(\mathbf{S}^{m-2}\right)$. There is a generalized version of the Whitney trick valid when $3 p<2 m$ due to Haefliger which can be used to regularly homotop $\mathbf{S}^{p}$ to an embedding. We wish to define an invariant $\sigma$ which at once is an obstruction to finding such a homotopy (for example consider the usual Whitney trick obstruction in the group ring) but also is insensitive to any movement of $\mathbf{S}^{m-2}$ in $\mathbf{S}^{m}-f\left(\mathbf{S}^{p}\right)$. Our invariant satisfies these criteria. Moreover, it is possible to construct link maps which realize nonzero values for $\sigma$.

The definition of $\sigma$ does not require the codimension two component to be a sphere. Thus we assume for this section that we are given a link map $f: \mathbf{S}^{p} \cup M^{m-2} \rightarrow \mathbf{S}^{m}$ where $M$ is any closed and oriented manifold. We continue to assume that $p \leq m-2$. Also, to avoid trivial complications, we will assume $p \geq 2$.

As pointed out in $\S 2, f$ can be perturbed slightly so that it immerses $\mathbf{S}^{p}$, and by the usual arguments we may assume that this immersion is self-transverse.

Let

$$
\widetilde{D}=\left\{(x, y) \in \mathbf{S}^{p} \times \mathbf{S}^{p} \mid f(x)=f(y) \text { and } x \neq y\right\}=(f \times f)^{-1}\left(\Delta_{\mathbf{S}^{m}}\right) .
$$

By transversality $\widetilde{D}$ is a manifold with a free $\mathbf{Z}_{2}$ action $(x, y) \mapsto(y, x)$. Let $D$ be the quotient manifold.

If $(x, y) \in \widetilde{D}$, let $\gamma$ be a path in $\mathbf{S}^{p}$ from $x$ to $y$. Then $f(\gamma)$ is a loop in $\mathbf{S}^{m}-f(M)$. The linking number $\operatorname{lk}(f(\gamma), f(M))$ is independent of the choice of $\gamma$ and hence the absolute value $|\operatorname{lk}(f(\gamma), f(M))|$ descends to a well-defined continuous function on $D$. In other words we have found a decomposition:

$$
D=\coprod_{k \geq 0} D_{k} \text {. }
$$

Each $D_{k}$ is a union of components of $D$. Let $\widetilde{D}_{k}$ be the inverse image of $D_{k}$ in $\widetilde{D}$.

Lemma 3.1. Let $k>0$. Then $\widetilde{D}_{k} \rightarrow D_{k}$ is a trivial double cover. Furthermore there is a canonical choice of first and second branch so that we can write $\widetilde{D}_{k}=$ $A_{k} \cup B_{k}$ with $A_{k} \cong B_{k} \cong D_{k}$.

Proof. Let $\Psi: \widetilde{D}_{k} \rightarrow \pm 1$ be given by $(x, y) \mapsto \frac{1}{k} \operatorname{lk}(f(\gamma), f(M))$, where $\gamma$ is a path from $x$ to $y$ in $\mathbf{S}^{p}$. Then $\Psi$ is continuous and so we write $A_{k}=\Psi^{-1}(1)$ and $B_{k}=\Psi^{-1}(-1)$. Clearly $(x, y) \in A_{k} \Leftrightarrow(y, x) \in B_{k}$.

Let $\operatorname{proj}_{i}: \mathbf{S}^{p} \times \mathbf{S}^{p} \rightarrow \mathbf{S}^{p}, i=1,2$, be the projections onto the first and second factors. Then generically $\operatorname{proj}_{i \mid A_{k}}$ is an immersion, and an embedding if $\frac{3}{2} p<m$. 
Now pick a point $\infty \in \mathbf{S}^{p}-\left(\operatorname{proj}_{1}\left(A_{k}\right) \cup \operatorname{proj}_{2}\left(A_{k}\right)\right)$. The normal bundle of $f, \nu_{f}$, admits a framing $\mathscr{F}=\left(v_{1}, \ldots, v_{m-p}\right)$ over $\mathbf{S}^{p}-\infty$. We can pull back this bundle over $A_{k}$ using the two immersions $\operatorname{proj}_{1 \mid A_{k}}$ and $\operatorname{proj}_{2 \mid A_{k}}$ to get two bundles which we denote by $\eta_{1}$ and $\eta_{2}$. The framing $\mathscr{F}$ pulls back to frame the $\eta_{i}$, and so by taking the sum of the framings we obtain a framing of $\eta_{1} \oplus \eta_{2}$.

It is obvious that this sum is the normal bundle of the immersion $f \circ$ $\operatorname{proj}_{1}: A_{k} \rightarrow \mathbf{S}^{m}$ : this is just the definition of transversality. Thus $A_{k}$ is in this way an immersed framed manifold in $\mathbf{S}^{m}$, so by the usual Thom-Pontryagin construction determines an element $\sigma_{k} \in \Omega_{2 p-m}^{f r}$.

The above construction does not work for $k=0$ since the general $\widetilde{D}_{0} \rightarrow D_{0}$ could be a nontrivial double cover. One might at first attempt to remedy this by defining $\sigma_{0}$ to be the double point Hopf invariant constructed by U. Koschorke and B. Sanderson in [KoS]. Unfortunately this is not a link homotopy invariant, although it gives the correct definition if we were to instead consider regular link homotopies.

Our solution is instead to define $\sigma_{0}=-\sum_{k>0} \sigma_{k}$. (Notice that only finitely many of the $\sigma_{k}$ are nonzero since only finitely many $D_{k}$ are nonempty.)

We now come to the definition of our invariant. Let $\Omega_{n}^{f r}[t]$ denote the formal sums $\sum_{i} a_{i} t^{i}$ where $a_{i} \in \Omega_{n}^{f r}$,

Definition 3.2. For $f: \mathbf{S}^{p} \cup M^{m-2} \rightarrow \mathbf{S}^{m}$ a link map as above, define

$$
\sigma=\sum_{k \geq 0} \sigma_{k} t^{k} \in \Omega_{2 p-m}^{f r}[t] .
$$

Theorem 3.3. $\sigma$ is a well-defined link homotopy invariant.

Proof. We will show that $\sigma_{k}$ is a link homotopy invariant for some fixed $k>0$.

First we need to see that $\sigma_{k}$ is independent of the choice of framing. Supposes then that $\mathscr{G}$ is some other framing on $\mathbf{S}^{p}-\infty$. Then the difference defines a map $\mathbf{S}^{p}-\infty \rightarrow \mathrm{SO}(m-p)$. Since $\mathbf{S}^{p}-\infty$ is contractible, it follows that this map is nullhomotopic. Equivalently there exists a one-parameter family of framings joining $\mathscr{F}$ to $\mathscr{G}$. Thus the framings on the bundles $\eta_{i}$ are homotopic and so they determine the same element in framed bordism.

Similarly, if we choose a different base point $\infty^{\prime}$, we can find an arc in $\mathbf{S}^{p}$ joining $\infty$ and $\infty^{\prime}$ which misses $\operatorname{proj}_{1}\left(A_{k}\right) \cup \operatorname{proj}_{2}\left(A_{k}\right)$ and the same argument works since again $\mathbf{S}^{p}-$ arc is contractible.

Next we show that $\sigma_{k}$ is unchanged if $f$ is altered by a link homotopy which is regular along $\mathbf{S}^{p}$. Given such a homotopy, $F$ say, we may take it to be selftransverse along $\mathbf{S}^{p}$, so that the double point set changes by a bordism. Even more is true: each $D_{k}$ changes by a bordism and there is no amalgamation of parts of $D_{k}$ with those of $D_{l}$ if $k \neq l$. Now a framing of $\nu_{f}$ over $\mathbf{S}^{p}-\infty$ extends to one of $\nu_{F}$ over $\left(\mathbf{S}^{p}-\infty\right) \times \mathbf{I}$, so that we can frame these bordisms. Thus $\sigma_{k}$ indeed is left unchanged. 
The next step is to show that the regular homotopy class of $f_{\mid \mathbf{S}^{p}}$ can be changed by a small perturbation without changing $\sigma_{k}$. Take a small sphere $\Sigma^{p}$ immersed in a ball close to $f\left(\mathbf{S}^{p}\right)$ and disjoint from $f(M)$ representing some regular homotopy class. Then the connected sum of $f\left(\mathbf{S}^{p}\right)$ and $\Sigma$ is obviously homotopic to $f\left(\mathbf{S}^{p}\right)$ in $\mathbf{S}^{m}-f(M)$. Since the self-intersection points of $\Sigma$ lie in a ball disjoint from $f(M)$, it follows that $D_{k}(\Sigma)=0$ for $k>0$, so that $D_{k}\left(f\left(\mathbf{S}^{p}\right)\right)=D_{k}\left(f\left(\mathbf{S}^{p}\right) \# \Sigma\right)$, and hence the new link map $\left(\mathbf{S}^{p} \# \Sigma\right) \cup M \rightarrow \mathbf{S}^{m}$ has the same $\sigma_{k}$-invariant as $f$. Since the collection of regular homotopy classes of immersions of $\mathbf{S}^{p}$ in $\mathbf{S}^{m}$ form a group, one can in particular choose $\Sigma$ to represent the inverse of $f_{\mid \mathbf{S}^{p}}$ and thus change $f$ by a link homotopy to a link map with $\mathbf{S}^{p}$ regularly homotopic to the standard embedding of $\mathbf{S}^{p} \subset \mathbf{S}^{m}$.

We now prove the general case. Let $f, g: \mathbf{S}^{p} \cup M \rightarrow \mathbf{S}^{p}$ be link homotopic maps which immerse $\mathbf{S}^{p}$. From the remarks of the preceding paragraph we can perturb $f$ and $g$ slightly so that $f_{\mid \mathbf{S}^{p}}$ and $g_{\mid \mathbf{S}^{p}}$ are regularly homotopic to the standard embedding without changing their $\sigma_{k}$. Now Theorem 2.4 implies that $f$ and $g$ are regularly link homotopic. But we have seen that $\sigma$ is unchanged by a regular link homotopy.

It is interesting to notice that $\sigma$ is also an invariant of singular link concordance, i.e. two link maps are identified if there is a map $F:\left(\mathbf{S}^{p} \cup \mathbf{S}^{m-2}\right) \times \mathbf{I} \rightarrow$ $\mathbf{S}^{m} \times \mathbf{I}$ (which is not necessarily level-preserving) such that $F\left(\mathbf{S}^{p} \times \mathbf{I}\right) \cap F\left(\mathbf{S}^{m-2}\right) \times$ $\mathbf{I}=\varnothing$. The proof follows the same lines. In contrast to the embedded theory where isotopy and concordance differ greatly, link homotopy and singular link concordance define the same equivalence classes in many dimensions [Ko3].

Proposition 3.4. 1. Let $f: \mathbf{S}^{p} \cup \mathbf{S}^{m-2} \rightarrow \mathbf{S}^{m}$ be a link map and $d: S^{m-2} \rightarrow S^{m-2}$ a degree $n$ map. Then

$$
\sigma(f \circ(1 \cup d))=(\operatorname{sign} n)^{m-p} \sum_{k>0} \sigma_{k}(f) \cdot\left(t^{k \cdot|n|}-1\right)
$$

2. If $g, f: \mathbf{S}^{p} \cup \mathbf{S}^{m-2} \rightarrow \mathbf{S}^{m}$ are link maps in different hemispheres, let $f \# g$ denote their connected sum along any embedded tubes. Then

$$
\sigma(f \# g)=\sigma(f)+\sigma(g) .
$$

3. Let $f: \mathbf{S}^{p} \cup \mathbf{S}^{m-2} \rightarrow \mathbf{S}^{m}$ be a link map and $r: S^{m} \rightarrow S^{m}$ a reflection. Then

$$
\sigma(r \circ f)=(-1)^{m-p+1} \sigma(f)
$$

Proof. 1. A loop $\gamma$ changing branches at one component of the double point set satisfies $\operatorname{lk}\left(\gamma, f \circ d\left(S^{m-2}\right)\right)=|n| \cdot \operatorname{lk}\left(\gamma, f\left(S^{m-2}\right)\right)$. If $d$ is negative, then the first and second branches of $S^{p}$ at a double point are reversed. Thus 

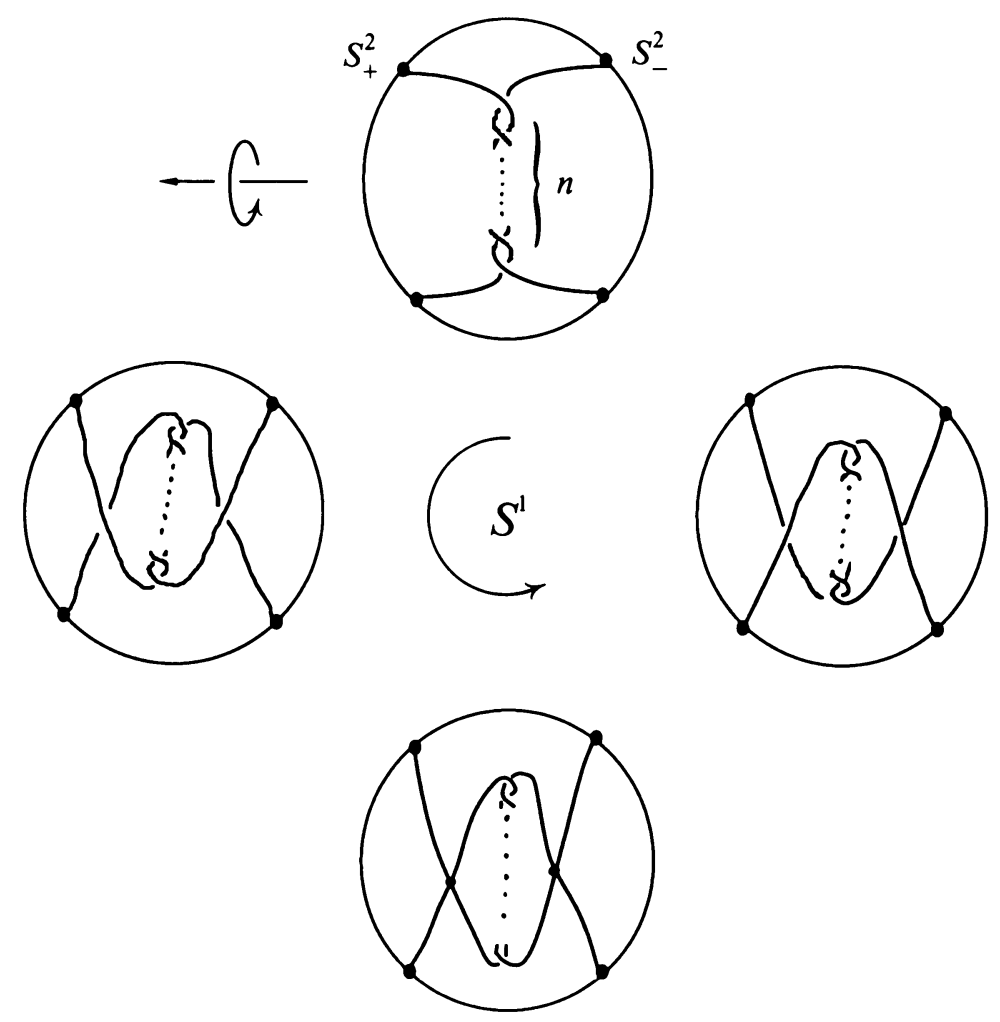

FIGURE 1

$\sigma_{k} \in \Omega_{2 p-m}^{f r}=\lim _{l \rightarrow \infty} \pi_{m+l}\left(\Sigma^{l} S^{2 m-2 p}\right)$ is changed to $h \circ \sigma_{k}$, where $h: S^{2 m-2 p} \rightarrow$ $S^{2 m-2 p}$ is the compactification of the map

$$
\begin{aligned}
\mathbf{R}^{2 m-2 p}=\mathbf{R}^{m-p} \times \mathbf{R}^{m-p} & \rightarrow \mathbf{R}^{m-p} \times \mathbf{R}^{m-p} \\
(x, y) & \rightarrow(y, x) .
\end{aligned}
$$

The degree of this map is $(-1)^{m-p}$.

2. The proof is obvious.

3. If $C$ is some twisted double point component of $S^{p} \rightarrow S^{m}$, then a degree -1 diffeomorphism of $S^{m}$ exchanges the first and second branches at $C$ since the sign of the linking number $1 \mathrm{k}\left(\gamma, f\left(S^{m-2}\right)\right)$ changes. ( $\gamma$ is a curve changing branches at $C$.)

Furthermore, the composite $S^{m} \stackrel{-1}{\longrightarrow} S^{m} \rightarrow S^{2 m-2 p}$ corresponds under the Thom-Pontryagin construction to reflecting the framed manifold. Hence, $\sigma_{k}(r \circ f)$ corresponds to

$$
S^{m} \stackrel{-1}{\longrightarrow} S^{m} \stackrel{\sigma_{k}(f)}{\longrightarrow} S^{2 m-2 p} \stackrel{(-1)^{m-p}}{\longrightarrow} S^{2 m-2 p} .
$$

(These are of course stable maps.) 
Corollary 3.5. The image of $\sigma: L M_{p, m-2}^{m} \rightarrow \Omega_{2 p-m}^{f r}[t]$ is either zero or an infinitely generated group.

The simplest examples to visualize are link maps $\mathbf{S}^{2} \cup \mathbf{S}^{2} \rightarrow \mathbf{S}^{4}$. Figure 1 shows an example of a link map with $\sigma=\left(t^{n}-1,1-t^{n}\right)$. Notice that if the intersection is zero dimensional, then the element of $\pi_{0}^{s}=\mathbf{Z}$ is just the sign of the intersection point. We are considering $S^{4}$ as $D^{3} \times S^{1} \cup S^{2} \times D^{2}$. An immersed $\mathbf{S}^{2}$ can be viewed as a regular homotopy of an interval in $\mathbf{D}^{3}$ to itself with boundary fixed, i.e. as a map of $\mathbf{I} \times \mathbf{S}^{1}$ in $\mathbf{D}^{3} \times \mathbf{S}^{1}$, which we "cap off" using $\mathbf{S}^{0} \times \mathbf{D}^{2}$ in $\mathbf{S}^{2} \times \mathbf{D}^{2}$.

Notice that there are two $\sigma$ invariants for a link map $f \in L M_{2,2}^{4}$, one for each component. It turns out that the value of each of these at zero is congruent mod two to the $\alpha$ invariant $\alpha \in \pi_{4} \mathbf{S}^{3}=\mathbf{Z}_{2}$. Thus $\sigma$ detects many nontrivial link homotopy classes that $\alpha$ does not, for example the connected sum of two of the link maps of Figure 1 has $\sigma=\left(2 t^{n}-2,2-2 t^{n}\right)$, which has $\alpha$ invariant zero.

We next describe a method for constructing link maps which realize nonzero values of $\sigma$. For certain values of $p, m$ we will be able to show that the image of $L M_{p, m-2}^{m}$ in $\Omega_{2 p-m}^{f r}[t]$ contains polynomials of arbitrarily high degree.

The first question which one can ask is whether there exist immersions $\mathbf{S}^{p} \rightarrow$ $\mathbf{S}^{m}$ such that some component of the double point manifold is nontrivially framed. At this point it is convenient to introduce the following definition.

Definition 4.1. Let $f: \mathbf{S}^{p} \rightarrow \mathbf{S}^{m}$ be a self-transverse immersion. A component $C$ of the double point manifold

$$
D=\left\{(x, y) \in \mathbf{S}^{p} \times \mathbf{S}^{p} \mid f(x)=f(y), x \neq y\right\} / \mathbf{Z}_{2}
$$

will be called twisted if

1. $C$ is trivially double covered by its inverse image in

$$
\widetilde{D}=\left\{(x, y) \in \mathbf{S}^{p} \times \mathbf{S}^{p} \mid f(x)=f(y), x \neq y\right\},
$$

2. If $\mathscr{F}$ is a framing of $\nu_{f}$ on $\mathbf{S}^{p}-\infty$ then $\mathscr{F}$ induces a framing on $C$ which is nonzero in $\Omega_{2 p-m}^{f r}$.

Notice that the element of $\Omega_{2 p-m}^{f r}$ is defined only up to sign since one must choose a first branch and second branch at $C$. Another remark is that a twisted component must be trivially double covered, this excludes what some might consider the most "twisted" types of self-intersections, but as we saw in the definition those components which are nontrivially double covered have no influence on the invariant $\sigma$.

Back to our question, then: Are there immersions $f: \mathbf{S}^{p} \rightarrow \mathbf{S}^{m}$ with some of the double point components twisted? A priori it may be that every component is trivially framed, so that $\sigma$ is always zero. 
We are grateful to Scott Carter for suggestions which led to the following lemma.

Lemma 4.2. Let $3 p+2 \leq 2 m$. Then for any $\alpha \in \operatorname{Im} J: \pi_{2 p-m} \mathrm{SO} \rightarrow \pi_{2 p-m}^{s}$, there exists an immersion $\mathbf{S}^{p} \rightarrow \mathbf{S}^{m}$ with double point manifold a twisted $(2 p-m)$ sphere representing $\alpha$.

Proof. Set $k=2 p-m$ and let $\mathbf{S}^{p-k} \rightarrow \mathbf{R}^{2 p-2 k}$ be an immersion with a single self-intersection point at the origin (see Whitney [W]). Choose a point $x$ in $S^{p-k}$ different from the two points which map to the origin. Given some $\alpha \in$ $\pi_{k} \mathrm{SO}_{2 p-2 k}, \alpha$ determines an immersion:

$$
\begin{aligned}
A: \mathbf{S}^{p-k} \times \mathbf{S}^{k} & \rightarrow \mathbf{R}^{2 p-2 k} \times \mathbf{S}^{k} \subset \mathbf{R}^{2 p-k} \\
(x, y) & \mapsto(x \cdot \alpha(y), y) .
\end{aligned}
$$

The double point manifold is a trivially double covered $k$-sphere $0 \times \mathbf{S}^{k}$ $\subset \mathbf{R}^{2 p-k}$. Its framing clearly represents $\alpha \in \pi_{k} \mathrm{SO}_{2 p-2 k}$. One checks that $\pi_{k} \mathrm{SO}_{2 p-2 k}=\pi_{k}$ SO if $4 p+2 \leq 3 m$.

Now the embedded sphere $x \times \mathbf{S}^{k} \subset \mathbf{S}^{p-k} \times \mathbf{S}^{k}$ is framed by choosing a small disk neighborhood of $x$ and spinning it around. To obtain an $\mathbf{S}^{p}$ from $\mathbf{S}^{p-k} \times \mathbf{S}^{k}$ we need to ambiently surger this sphere. It bounds an embedded $\mathbf{D}^{k+1}$ which misses the immersed $\mathbf{S}^{p-k} \times \mathbf{S}^{k}$ except along the boundary since $(k+1)+p<2 p-k$ (this is where we need $3 p<2 m$ ). The framing along the boundary extends to the disk. In fact, the coordinates given by the natural framing of the disk allow us to construct a difference map $\mathbf{S}^{k} \rightarrow V_{p-k, 2 p-2 k-1}$ along the boundary. But $V_{p-k, 2 p-2 k-1}$ is $p-k-2$-connected, and $k \leq p-k-2$. Thus the framing on the boundary extends to a framing on the disc and so we can complete the surgery.

This construction also works to construct an immersion $\mathbf{S}^{3} \rightarrow \mathbf{S}^{5}$ with double point set a circle which is trivially double covered and nontrivially framed. One must use more care to find a 2-disk for the surgery which misses the $S^{2} \times S^{1}$. It is also necessary to keep closer track of the framing. For details see [Hu].

We will now describe an "algorithm" to produce link maps with nonzero $\sigma$. Let $f_{1}: \mathbf{S}^{p} \rightarrow \mathbf{S}^{m}$ be a self-transverse immersion with only one double point component and assume this component is twisted; so it represents $\pm \beta \in \Omega_{2 p-m}^{f r}$. Assume also that the immersion has no triple points. The immersions produced by Lemma 4.2 satisfy these criteria.

The image $f_{1}\left(\mathbf{S}^{p}\right)$ is homotopy equivalent to $\mathbf{S}^{p} \cup_{C \times \partial I} C \times \mathbf{I}$, where $C$ is this component. Computing with the Mayer-Vietoris sequence one gets $H_{1}\left(f_{1}\left(\mathbf{S}^{p}\right)\right)=\mathbf{Z}$; a generator is given by a curve which changes branches at some point in $C$. 
Pick an immersion $f_{2}: \mathbf{S}^{m-2} \rightarrow \mathbf{S}^{m}-f\left(\mathbf{S}^{p}\right)$. The following diagram commutes (A.D. denotes the Alexander duality isomorphisms):

$$
\begin{array}{ccc}
H_{m-2}\left(f_{2}\left(\mathbf{S}^{m-2}\right)\right) & \longrightarrow H_{m-2}\left(\mathbf{S}^{m}-f_{1}\left(\mathbf{S}^{p}\right)\right) \\
\partial \uparrow \cong & \partial \uparrow \cong \\
H_{m-1}\left(\mathbf{S}^{m}, f_{2}\left(\mathbf{S}^{m-2}\right)\right) & \longrightarrow & H_{m-1}\left(\mathbf{S}^{m}, f_{1}\left(\mathbf{S}^{p}\right)\right) \\
\text { A.D. } \uparrow \cong & \text { A.D. } \uparrow \cong \\
H^{1}\left(\mathbf{S}^{m}-f_{2}\left(\mathbf{S}^{m-2}\right)\right) & \longrightarrow & H^{1}\left(f_{1}\left(\mathbf{S}^{p}\right)\right)
\end{array}
$$

So the homomorphisms

$$
H_{1}\left(f_{1}\left(\mathbf{S}^{p}\right)\right) \rightarrow H_{1}\left(S^{m}-f_{2}\left(\mathbf{S}^{m-2}\right)\right)
$$

and

$$
H_{m-2}\left(f_{2}\left(\mathbf{S}^{m-2}\right)\right) \rightarrow H_{m-2}\left(\mathbf{S}^{p}-f_{1}\left(\mathbf{S}^{p}\right)\right)
$$

are algebraically dual.

Since $f_{2}\left(\mathbf{S}^{m-2}\right)$ is an $(m-2)$-sphere with identifications along a complex of dimension $\leq m-4$, the homomorphism $H_{m-2}\left(\mathbf{S}^{m-2}\right) \rightarrow H_{m-2}\left(f_{2}\left(\mathbf{S}^{m-2}\right)\right)$ is an isomorphism. If the Hurewicz homomorphism

$$
\pi_{m-2}\left(\mathbf{S}^{m}-f_{1}\left(\mathbf{S}^{p}\right)\right) \rightarrow H_{m-2}\left(\mathbf{S}^{m}-f_{1}\left(\mathbf{S}^{p}\right)\right)
$$

is nonzero, pick an immersion which represents a nonzero homology class. Then the horizontal homomorphisms in the commutative diagram above are nonzero, from which it follows that the homomorphism

$$
\mathbf{Z}=H_{1}\left(f_{1}\left(\mathbf{S}^{p}\right)\right) \rightarrow H_{1}\left(\mathbf{S}^{m}-f_{2}\left(\mathbf{S}^{m-2}\right)\right)=\mathbf{Z}
$$

is multiplication by some nonzero integer $k$. A glance at the definition of $\sigma$ will convince the reader that the link map $f_{1} \cup f_{2}-f: \mathbf{S}^{p} \cup \mathbf{S}^{m-2} \rightarrow \mathbf{S}^{m}$ has $\sigma(f)= \pm \beta \cdot\left(t^{|k|}-1\right)$. (The sign depends on the sign of $k$.)

An understanding of the nontriviality of $\sigma$, therefore, depends first on finding immersions with twisted double point components (such as those given in Lemma 4.2) and then showing that the Hurewicz map in dimension $m-2$ is nontrivial on the complement of such an immersion. This is the approach we will take in the examples below.

If one just wants a nonspherical codimension two component, it would of course suffice to show that $\Omega_{m-2}\left(\mathbf{S}^{m}-f\left(\mathbf{S}^{p}\right)\right) \rightarrow H_{m-2}\left(\mathbf{S}^{m}-f\left(\mathbf{S}^{p}\right)\right)$ is nonzero. This is often much easier to show, using for example the Atiyah-Hirzebruch spectral sequence. An easy argument shows that this homomorphism is onto if $3 p \leq 2 m-4$, so using Lemma 4.2 we can conclude that there exist link maps $f: \mathbf{S}^{p} \cup M^{m-2} \rightarrow \mathbf{S}^{m}$ with $\sigma(f)$ any polynomial

$$
\sum_{i>0} a_{i}\left(t^{i}-1\right) \text { for } a_{i} \in \operatorname{Im} J
$$

Example. $L M_{p, 2 p-2}^{2 p}$ for $p>2$. 
Note that $p$ is middle dimensional. Thus $\sigma$ takes values in $\mathbf{Z}[t]$. The image of $\sigma$ is calculated in the following theorem.

Theorem 4.3. 1. If $p=4$ or 8 then the image of $\sigma: L M_{p, 2 p-2}^{2 p} \rightarrow \Omega_{0}^{f r}[t]=\mathbf{Z}[t]$ is the subgroup $\{p(t) \mid p(1)=0\}$.

2. If $p$ is even, $p \neq 4$ or 8 , then the image of $\sigma$ is

$$
\left\{p(t) \mid p(1)=0 \text { and } d p / d t_{\mid t=1} \equiv 0 \bmod 2\right\} \text {. }
$$

3. If $p$ is odd then the image of $\sigma$ is

$$
\left\{p(t) \mid p(1)=0 \text { and } d p / d t_{\mid t=1}=0\right\} .
$$

The conclusion of 1 holds also when $p=2$ (see [Kil]). Notice that $p(1)=0$ always so that for $p=4$ or 8 the image is as large as it can be. The second (respectively third) parts of this theorem can be reworded as $\sum_{k>0} k \cdot \sigma_{k}$ is congruent to zero mod 2 (resp. is equal to zero).

The next fact will be used in proving Theorem 4.3. Recall that a selftransverse immersion $\mathbf{S}^{p} \rightarrow \mathbf{S}^{2 p}$ has isolated self-intersection points.

Proposition 4.4. If $\mathbf{S}^{p} \rightarrow \mathbf{S}^{2 p}, p>2$, is a self-transverse immersion with $n$ double point, then $\mathbf{S}^{2 p}-f\left(\mathbf{S}^{p}\right)$ is homotopy equivalent to $\mathbf{S}^{p-1} \bigcup_{i=1}^{n} e_{i}^{2 p-2}$ where the cells $e_{i}$ are attached by $\pm\left[i_{p-1}, i_{p-1}\right] . \quad\left(\left[i_{p-1}, i_{p-1}\right] \in \pi_{2 p-3} \mathbf{S}^{p-1}\right.$ denotes the Whitehead product.)

Proof. Let $X=\mathbf{S}^{2 p}-f\left(\mathbf{S}^{p}\right) . X$ is $(p-2)$-connected, and $\pi_{p-1} X=H_{p-1} X=$ $H^{p}\left(f\left(\mathbf{S}^{p}\right)\right)=\mathbf{Z}$ by Alexander duality. The generator is represented by an embedded meridian sphere $\mathbf{S}_{\mu}^{p-1} \subset X$. Then $H_{i}\left(X, \mathbf{S}_{\mu}^{p-1}\right)=H_{i} X$ for $i \geq p$. Using Alexander duality again we compute that $H_{i}\left(X, \mathbf{S}_{\mu}^{p-1}\right)$ vanishes for $i \leq 2 p-3$ and $H_{2 p-2}\left(X, \mathbf{S}_{\mu}^{p-1}\right)=H_{2 p-2} X=H^{1}\left(f\left(\mathbf{S}^{p}\right)\right)=\mathbf{Z}^{n}$. The last equality follows from the fact that $f\left(\mathbf{S}^{p}\right)$ has the homotopy type of $\mathbf{S}^{p} \vee_{i} \mathbf{S}_{i}^{1}$.

The relative Hurewicz theorem now implies that

$$
\pi_{2 p-2}\left(X, \mathbf{S}_{\mu}^{p-1}\right)=H_{2 p-2}\left(X, \mathbf{S}_{\mu}^{p-1}\right) .
$$

Consider the commutative diagram:

$$
\begin{gathered}
\pi_{2 p-2} \mathbf{S}_{\mu}^{p-1} \rightarrow \pi_{2 p-2} X \quad \rightarrow \quad \pi_{2 p-2}\left(X, \mathbf{S}_{\mu}^{p-1}\right) \rightarrow \pi_{2 p-3} \mathbf{S}_{\mu}^{p-1} \\
H_{2 p-2} X \stackrel{\downarrow \cong}{\cong} H_{2 p-2}\left(X, \mathbf{S}_{\mu}^{p-1}\right)
\end{gathered}
$$

The generators of $\mathrm{H}_{2 p-2} X$ are represented by "linking-tori". These are embedded $\mathbf{S}^{p-1} \times \mathbf{S}^{p-1}$, one for each self-intersection point. More precisely let $x, y \in \mathbf{S}^{p}$ be a pair with $f(x)=f(y)$. Let $\mathbf{S}_{\varepsilon}^{p-1} \subset \mathbf{S}^{p}$ be a sphere which separates $x$ from all the other double points. A neighborhood of $\mathbf{S}_{\varepsilon}^{p-1}$ is embedded by $f$ and the linking torus for $f(x)=f(y)$ is the boundary of the normal bundle of $\mathbf{S}^{p}$ restricted to $\mathbf{S}_{\varepsilon}^{p-1}$. An easy argument using Alexander duality shows that indeed these linking tori generate $\mathrm{H}_{2 p-2} \mathrm{X}$. 
For each linking torus $\mathbf{S}^{p-1} \times \mathbf{S}^{p-1}$, both $\mathbf{S}^{p-1} \times *$ and $* \times \mathbf{S}^{p-1}$ are freely homotopic to the fixed meridian $\mathbf{S}_{\mu}^{p-1}$ (at least up to sign). It follows now that $\pi_{2 p-2}\left(X, \mathbf{S}_{\mu}^{p-1}\right)$ is generated by maps $\left(\mathbf{D}^{2 p-2}, \mathbf{S}^{2 p-3}\right) \rightarrow\left(X, \mathbf{S}_{\mu}^{p-1}\right)$ such that the restriction to $\mathbf{S}^{2 p-3}$ represents the Whitehead product (up to sign). Using this description, then, one can map $\mathbf{S}^{p-1} \bigcup_{i=1}^{n} e_{i}^{2 p-2}$ to $X$ inducing a homology equivalence. The proposition now follows from the Whitehead theorem.

Proof of Theorem 4.3. 1. Suppose $p=4$ or 8. It suffices to show that there exists a link map with $\sigma= \pm(t-1)$ since starting with such a link map and using the three operations of Proposition 3.4 one can generate the entire set $\{p(t) \mid p(1)=0\}$.

Let $f_{1}: \mathbf{S}^{p} \rightarrow \mathbf{S}^{2 p}$ be an immersion with a single self-intersection point $x$. Let $\gamma_{x}$ be a loop in $f_{1}\left(\mathbf{S}^{p}\right)$ which changes branches at $x ; \gamma_{x}$ generates $H_{1}\left(f_{1}\left(\mathbf{S}^{p}\right)\right)$.

The Whitehead product $\left[i_{p-1}, i_{p-1}\right]$ is zero in these dimensions, so $X=$ $\mathbf{S}^{p-1} \vee \mathbf{S}^{2 p-2}$. Thus $\pi_{2 p-2} X \rightarrow H_{2 p-2} X$ is onto. Pick a map $f_{2}: \mathbf{S}^{2 p-2} \rightarrow X$ which represents a generator of $H_{2 p-2} X=\mathbf{Z}$. Then, as we have seen above,

$$
\mathbf{Z}=H^{1}\left(f_{1}\left(\mathbf{S}^{p}\right)\right) \rightarrow H^{1}\left(\mathbf{S}^{2 p}-f_{2}\left(\mathbf{S}^{2 p-2}\right)\right)=\mathbf{Z}
$$

is onto, and hence is an isomorphism. Therefore

$$
H_{1}\left(\mathbf{S}^{2 p}-f_{2}\left(\mathbf{S}^{2 p-2}\right)\right) \rightarrow H_{1}\left(f_{1}\left(\mathbf{S}^{p}\right)\right)
$$

is also an isomorphism. Hence $\operatorname{lk}\left(\gamma_{x}, f_{2}\left(\mathbf{S}^{2 p-2}\right)\right)= \pm 1$. The point $x$ is "framed" so that $\sigma\left(f_{1} \cup f_{2}\right)= \pm(t-1)$.

2. If $p$ is even but not equal to 4 or 8 , the Whitehead product $\left[i_{p-1}, i_{p-1}\right]$ has order 2. Given any self-transverse immersion $f: \mathbf{S}^{p} \rightarrow \mathbf{S}^{2 p}$, let $X=\mathbf{S}^{2 p}$ $f\left(\mathbf{S}^{p}\right)$. The linking tori determine generators $z_{x} \in H_{2 p-2} X$, one for each double point $x$. If $\gamma_{2}$ is a loop in $f\left(\mathbf{S}^{p}\right)$ changing branches at $x$, then $\operatorname{lk}\left(\gamma_{x}, z_{x}\right)=$ \pm 1 . The commutative diagram of Proposition 5.6 gives an exact sequence:

$$
\pi_{2 p-2} X \stackrel{h}{\longrightarrow} H_{2 p-2} X \stackrel{\partial}{\longrightarrow} \mathbf{Z}_{2} \rightarrow 0 .
$$

Moreover the proof of the proposition shows $\partial\left(z_{x}\right)=\left[i_{p-1}, i_{p-1}\right]=1 \in \mathbf{Z}_{2}$. Thus a class $\sum a_{x} \cdot z_{x}$ is spherical if and only if $\sum a_{x} \equiv 0(\bmod 2)$.

Since a link map $f: \mathbf{S}^{p} \cup \mathbf{S}^{2 p-2} \rightarrow \mathbf{S}^{2 p}$ determines a spherical element, the homomorphism

$$
\mathbf{Z}=H_{2 p-2}\left(f\left(\mathbf{S}^{2 p-2}\right)\right) \rightarrow H_{2 p-2} X=\mathbf{Z}^{m}
$$

must take the generator to $\sum a_{x} z_{x}$ with $\sum a_{x} \equiv 0 \bmod 2$ (where $m$ is the number of double points). This implies that the homomorphism

$$
H_{1}\left(f\left(\mathbf{S}^{p}\right)\right) \rightarrow H_{1}\left(\mathbf{S}^{2 p}-f\left(\mathbf{S}^{2 p-2}\right)\right)=\mathbf{Z}
$$

takes the class $\left[\gamma_{x}\right]$ to an integer $a_{x}$ so that $\sum a_{x} \equiv 0 \bmod 2$.

But $a_{x}=\operatorname{lk}\left(\gamma_{x}, f\left(\mathbf{S}^{2 p-2}\right)\right)$. One sees from the definition of $\sigma_{k}$ that $\sum_{k>0} k$. $\sigma_{k} \equiv 0 \bmod 2$. Conversely, given a class $\sum a_{x} z_{x} \in H_{m-2} X$ with $\sum a_{x} \equiv$ 
$0 \bmod 2$, this class can be represented by a map $\mathbf{S}^{2 p-2} \rightarrow X$. The resulting link map has $\sigma \equiv b+\sum t^{\left|a_{x}\right|} \bmod 2$, so $d \sigma / d t_{\mid t=1} \equiv 0 \bmod 2$. (2) now follows immediately.

3. This case is similar to case 2 except that one must be more careful with orientations. The essential point here is that $\left[i_{p-1}, i_{p-1}\right]$ has infinite order. One then makes use of the exact sequence

$$
\pi_{2 p-2} X \stackrel{h}{\longrightarrow} H_{2 p-2} X \stackrel{\partial}{\longrightarrow} \mathbf{Z} \rightarrow 0 .
$$

We omit the details.

One can obtain an alternative proof of a part of this result, namely that $d \sigma /\left.d t\right|_{t=1}$ must satisfy the conditions in Theorem 4.5, by using the work of U. Koschorke in [Ko2]. Let $G=\mathbf{Z}$ or $\mathbf{Z}_{2}$ according to whether $p$ is odd or even. An easy argument shows that the homomorphism

$$
\mathbf{Z}[t]=\Omega_{0}^{f r}[t] \rightarrow \Omega_{0}\left(R P^{\infty},(p-1) \lambda\right)=G
$$

given by $p(t) \mapsto d p /\left.d t\right|_{t=1}$ takes $\sigma$ to Koschorke's invariant $\beta$. Koschorke proves that there is a commutative diagram

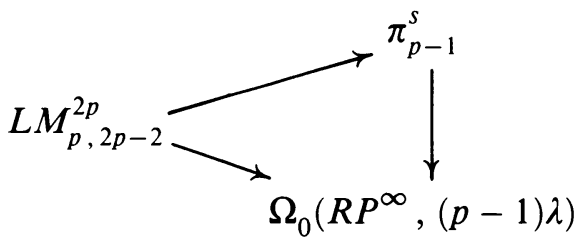

where the vertical arrow is the stable Hopf invariant. This map is zero except when $p=2,4$ or 8 . But this fact implies that the image of $\sigma$ is contained in the sets described in Theorem 4.3.

Having determined the image of $\sigma: L M_{p, m-2}^{m} \rightarrow \mathbf{Z}[t]$, we now turn to a study of its kernel.

Theorem 4.5. Let $f: \mathbf{S}^{p} \cup \mathbf{S}^{2 p-2} \rightarrow \mathbf{S}^{2 p}$, with $p>2$. Then $\sigma=0$ if and only if $f$ is link homotopic to a link map which embeds $\mathbf{S}^{p}$.

Proof. If $f$ is link homotopic to a map which embeds $\mathbf{S}^{p}$, then clearly $\sigma=0$. So assume $\sigma=0$.

Perturb $f_{\mid \mathbf{S}^{p}}$ so that it is regularly homotopic to the standard embedding (in $\left.\mathbf{S}^{2 p}\right)$. For each double point $x$ let $\gamma_{x}$ be a loop in $f\left(\mathbf{S}^{p}\right)$ changing branches at $x$ and with $n_{x}=\operatorname{lk}\left(\gamma_{x}, f\left(\mathbf{S}^{2 p-2}\right)\right) \geq 0$. Since each $\sigma_{k}=0$ the double points of $f\left(\mathbf{S}^{p}\right)$ come in paits $(x, y)$ with opposite framings and with $n_{x}=$ $n_{y}$. This data would be sufficient to remove the self-intersections of $f\left(\mathbf{S}^{p}\right)$ if $\pi_{i}\left(\mathbf{S}^{2 p}-f\left(\mathbf{S}^{2 p-2}\right)\right)=\mathbf{Z}$, since $\sigma$ is equivalent to the usual obstruction in $\mathbf{Z}[\pi]$. Take the Whitney discs given by the data and note that although these discs may intersect $f\left(\mathbf{S}^{2 p-2}\right)$ in their interior, the algebraic intersection of each disc with $f\left(\mathbf{S}^{2 p-2}\right)$ is zero. Thus we can move $f\left(\mathbf{S}^{2 p-2}\right)$ off these Whitney discs (perhaps 
increasing the self-intersections of $f\left(\mathbf{S}^{2 p-2}\right)$ in the process). Now $\mathbf{S}^{p}$ can be regularly homotoped to an embedding in the complement of $f\left(\mathbf{S}^{2 p-2}\right)$.

We now have enough information available to decide when a link map $f: \mathbf{S}^{p} \cup$ $\mathbf{S}^{2 p-2} \rightarrow \mathbf{S}^{2 p}$ is nullhomotopic (except for a $\mathbf{Z}_{2}$ factor when $p$ is odd):

Theorem 4.6. Let $f: \mathbf{S}^{p} \cup \mathbf{S}^{2 p-2} \rightarrow \mathbf{S}^{2 p}$ be a link map with $p>2$. Then if $p$ is even or $p=3,7$, then $f$ is link-homotopically trivial if and only if both $\sigma$ and $\alpha$ are both zero. If $p$ is odd and both $\alpha$ and $\sigma$ vanish, then there is an element in $\mathbf{Z}_{2}$ whose vanishing implies that $f$ is link-homotopically trivial.

Proof. If $\sigma=0$ then $f$ is link homotopic to a map which embeds $\mathbf{S}^{p}$. Then the linking coefficient $\lambda \in \pi_{2 p-2} \mathbf{S}^{p-1}$ given by $\mathbf{S}^{2 p-2} \stackrel{f}{\longrightarrow} \mathbf{S}^{2 p}-\mathbf{S}^{p} \sim \mathbf{S}^{p-1}$ is defined, and the results of [Ki2] show that $f$ can be changed by a link homotopy to replace $\lambda$ by $\lambda+\gamma$ for any $\gamma \in \operatorname{ker} \Sigma: \pi_{2 p-2} \mathbf{S}^{p-1} \rightarrow \pi_{2 p-1} \mathbf{S}^{p}$, so that $f$ is nullhomotopic if $\Sigma \lambda(f)=0$. The group $\pi_{2 p} \mathbf{S}^{p+1}$ is the first stable group and so from Theorem 2.2 we see that $\Sigma^{2} \lambda=\alpha$. Computing with the EHP sequence shows that

$$
\Sigma: \Sigma\left(\pi_{2 p-2} \mathbf{S}^{p-1}\right) \rightarrow \pi_{2 p} \mathbf{S}^{p+1}=\pi_{p-1}^{s}
$$

is injective if $p$ is even or $p=3,7$ and has kernel $\mathbf{Z}_{2}$ otherwise. This means that $\Sigma \lambda(f)=0$ if and only if $\alpha(f)=0$ when $p$ is even or $p=5,7$. When $p$ is odd one could have $\Sigma \lambda(f) \neq 0$ but $\alpha(f)=0$. Since all elements of

$$
\text { Image } \Sigma^{\infty}: \pi_{2 p-2} \mathbf{S}^{p-1} \rightarrow \pi_{p-1}^{s}
$$

can be realized by link maps by Theorem 2.2 , this extra $Z_{2}$ can be realized by a link map.

Corollary 4.7. If $p$ is even, $p \geq 4$, then

$$
\alpha \oplus \sigma: L M_{p, 2 p-2}^{2 p} \rightarrow \text { Image } \alpha \oplus \mathbf{Z}^{\infty}
$$

is bijective, where $\mathbf{Z}^{\infty} \subset \mathbf{Z}[t]$ is the image of $\sigma$ given by Theorem 4.5. Furthermore, $\alpha$ is surjective for $p \neq 4,8$, and has index at most 2 for $p=4$ or 8.

Proof. The statements about Image $\alpha$ follows straightforwardly from the EHP sequence. The proof now follows from the observation that $\alpha \oplus \sigma$ is additive, together with Proposition 2.6.

It follows from recent work by $U$. Koschorke [Ko4] that this extra $Z_{2}$ in Theorem 4.6 is indeed a link homotopy obstruction. Thus Corollary 4.7 extends to the case of $p$ odd if we use $\Sigma \lambda$ instead of $\alpha$. In other words,

$$
\Sigma \lambda \oplus \sigma: L M_{p .2 p-2}^{2 p} \rightarrow \text { Image } \Sigma \lambda \oplus \mathbf{Z}^{\infty}
$$

is a bijection, where $\mathbf{Z}^{\infty}$ is given by Theorem 4.3 and Image $\Sigma \lambda \in \pi_{3 p-1} \mathbf{S}^{2 p}$. 
Example. $L M_{p, 2 p-3}^{2 p}$ when $p>3$.

We mentioned at the beginning of this section that constructing examples of link maps with nonzero $\sigma$ depended on showing that the Hurewicz map $\pi_{m-2}\left(\mathbf{S}^{m}-f\left(\mathbf{S}^{p}\right)\right) \rightarrow H_{m-2}\left(\mathbf{S}^{m}-f\left(\mathbf{S}^{p}\right)\right)$ is nonzero for "good" immersions $f: \mathbf{S}^{p} \rightarrow \mathbf{S}^{m}$. This becomes increasingly difficult as $p$ increases beyond $\frac{m}{2}$ since the homotopy groups become more difficult to manage. In this section we show that elements in $L M_{p, 2 p-3}^{2 p-1}$ can be detected using $\sigma$. The proof amounts to showing that the Hurewicz map is nontrivial for the immersions given by Lemma 4.2 .

In this dimension $\sigma$ takes values in $\Omega_{1}[t]=\mathbf{Z}_{2}[t]$.

Proposition 4.8. There exists a link map $f: \mathbf{S}^{p} \cup \mathbf{S}^{2 p-3} \rightarrow \mathbf{S}^{2 p-1}$ with $\sigma(f)=$ $t^{n}+1$, where $n$ is a positive integer dividing the order of $\pi_{2 p-4} \mathbf{S}^{p-2}$. Moreover, the image of $\sigma$ is an infinitely generated subgroup of $\mathbf{Z}_{2}[t]$.

Proof. The second statement follows from the first using Corollary 3.5.

Fix an immersion $f: \mathbf{S}^{p} \rightarrow \mathbf{S}^{m}$ with a single $t$ wisted circle as the double point manifold. Such an immersion is provided by Lemma 4.2

$f\left(\mathbf{S}^{p}\right)$ is homotopy equivalent to $\mathbf{S}^{p} \cup_{\mathbf{S}^{1} \times \partial I} \mathbf{S}^{1} \times \mathbf{I}$, and so one computes using the Mayer-Vietoris sequence:

$$
H_{i}\left(f\left(\mathbf{S}^{p}\right)\right)= \begin{cases}\mathbf{Z} & \text { if } i=1 \text { or } 2, \\ H_{i} \mathbf{S}^{p} & \text { if } i>2 .\end{cases}
$$

The generator of $H_{1}\left(f\left(\mathbf{S}^{p}\right)\right)$ is given by the loop changing branches at some point in the double point circle.

Now let $X=\mathbf{S}^{2 p-1}-f\left(\mathbf{S}^{p}\right)$ and let $\mathbf{S}_{\mu}^{p-2} \subset X$ be a meridian sphere. Then using Alexander duality and the computation above we see

$$
H_{i}\left(X, \mathbf{S}_{\mu}^{p-2}\right)= \begin{cases}0 & \text { if } i \leq 2 p-5 \\ \mathbf{Z} & \text { if } i=2 p-4 \text { or } 2 p-3 .\end{cases}
$$

The relative Hurewicz theorem implies that

$$
\pi_{i}\left(X, \mathbf{S}_{\mu}^{p-2}\right)=H_{i}\left(X, \mathbf{S}_{\mu}^{p-2}\right)
$$

for $i \leq 2 p-4$. The Blakers-Massey theorem implies that $\pi_{i}\left(X, \mathbf{S}_{\mu}^{p-2}\right)=$ $\pi_{i}\left(X / \mathbf{S}_{\mu}^{p-2}\right)$ for $i \leq 2 p-3$ and then we can apply a theorem of $\mathrm{G}$. Whitehead to conclude that the sequence

$$
\pi_{2 p-3}\left(X, \mathbf{S}_{\mu}^{p-2}\right) \rightarrow H_{2 p-3}\left(X, \mathbf{S}_{\mu}^{p-2}\right) \rightarrow H_{2 p-3}(\mathbf{Z}, 2 p-4) \rightarrow 0
$$

is exact. (This is easily proved using the spectral sequence for the fibration $\left.F \rightarrow X / \mathbf{S}_{\mu}^{p-2} \rightarrow K(\mathbf{Z}, 2 p-4)\right)$. But $H_{2 p-3}(\mathbf{Z}, 2 p-4)=0$. Consider the commuting diagram:

$$
\begin{gathered}
\pi_{2 p-3}\left(\mathbf{S}_{\mu}^{p-2}\right) \rightarrow \pi_{2 p-3} X \rightarrow \pi_{2 p-3}\left(X, \mathbf{S}_{\mu}^{p-2}\right) \rightarrow \pi_{2 p-4} \mathbf{S}_{\mu}^{p-2} \\
\downarrow \\
\mathbf{Z}=H_{2 p-3} X \stackrel{\downarrow}{\cong} H_{2 p-3}\left(X, \mathbf{S}_{\mu}^{p-2}\right)
\end{gathered}
$$


Clearly if $n=\operatorname{order} \pi_{2 p-4} \mathbf{S}_{\mu}^{p-2}$, there exists a map $f: \mathbf{S}^{2 p-3} \rightarrow X$ representing $n \in H_{2 p-3} x=\mathbf{Z}$. As we have seen before this implies that the map

$$
\mathbf{Z}=H_{1}\left(f\left(\mathbf{S}^{p}\right)\right) \rightarrow H_{1}\left(\mathbf{S}^{2 p-1}-f\left(\mathbf{S}^{2 p-3}\right)\right)=\mathbf{Z}
$$

takes the generating loop to $\pm n$. Equivalently, this loop links the codimension two component $n$ times. Thus the link map $f: \mathbf{S}^{p} \cup \mathbf{S}^{2 p-3} \rightarrow \mathbf{S}^{2 p-1}$ has $\sigma_{n}=$ $1 \in \mathbf{Z}_{2}=\Omega_{1}^{f r}$, so that $\sigma(f)=t^{n}+1 \in \mathbf{Z}_{2}[t]$.

It should be possible to continue this sort of analysis to get results in a few more dimensions. Unfortunately, this method will not work for pure codimension two links maps since the complements are not simply connected.

To illustrate the usefulness of $\sigma$ we will now prove that if $f: \mathbf{S}^{p} \cup \mathbf{S}^{m-2} \rightarrow \mathbf{S}^{m}$ is a link map which embeds the codimension two components, then $f$ is link homotopically trivial in the metastable range. Although $\sigma$ does not appear in the statement of this theorem, we will show that $\sigma$ is the only obstruction to nullhomotoping a link map with the codimension two component embedded, then we will show that $\sigma$ vanishes.

Theorem 5.1. Let $f: \mathbf{S}^{p} \cup \mathbf{S}^{m-2} \rightarrow \mathbf{S}^{m}$ be a link map which embeds the codimension two component. If $3 p+4 \leq 2 m$ and $m \geq 6$ then $f$ is link homotopically trivial.

In this theorem it is essential that the codimension two component is a sphere. Of course if $2 p \leq m-1$, then the $p$-sphere embeds automatically. It then follows from the main theorem of [Ki2] that such a link is nullhomotopic. So we will assume for the rest of this section that $2 p \geq m$.

We can outline the proof in the following way: using a cobordism argument one finds a homotopy of $\mathbf{S}^{m-2}$ in $\mathbf{S}^{m}-f\left(\mathbf{S}^{p}\right)$ to another embedding which has a highly connected universal cover. Then $\sigma$ will be seen to be the only obstruction to homotoping all the translates of liftings of $f: \mathbf{S}^{p} \rightarrow \mathbf{S}^{m}-\mathbf{S}^{m-2}$ disjoint embeddings, and hence $f$ is itself link homotopic to an embedded link if $\sigma(f)$ vanishes. Then we show that $\sigma$ vanishes. Finally, using the results of [Ki2] one concludes that $f$ is link homotopically trivial.

Lemma 5.2. Let $f: \mathbf{S}^{p} \cup \mathbf{S}^{m-2} \rightarrow \mathbf{S}^{m}$ embed the codimension 2 component. Suppose $m \geq 6$ and $p \leq m-3$. Then $\mathbf{S}^{m-2}$ is homotopic in $\mathbf{S}^{m}-f\left(\mathbf{S}^{p}\right)$ to another embedding so that

$$
\pi_{i}\left(\mathbf{S}^{m}-\mathbf{S}^{m-2}\right) \cong \pi_{i} \mathbf{S}^{1}, \quad i \leq m-p-2 .
$$

Proof. Perturb $f$ so that $f_{\mid \mathbf{S}^{p}}$ is a self-transverse immersion. We think of $f\left(\mathbf{S}^{p}\right)$ as an embedded $p$-dimensional subcomplex of $\mathbf{S}^{m}$. Let $U$ be a regular neighborhood of $f\left(\mathbf{S}^{p}\right)$ and $V$ a regular neighborhood of $f\left(\mathbf{S}^{m-2}\right)$.

Denote $\mathbf{S}^{m}-V$ by $X_{1}$. Since $f$ embeds $\mathbf{S}^{m-2}, H_{*} X_{1}=H_{*} \mathbf{S}^{1}$ and $\pi_{1} X_{1}$ is normally generated by any meridian circle. 
Attach 2-handles to $X_{1} \times \mathbf{I}$ to abelianize its fundamental group. The handles can be attached along nullhomologous circles in $X_{1}-f\left(\mathbf{S}^{p}\right)$ since $p+1<m$. Call the resulting bordism $W_{2}$ and write $\partial W_{2}=X_{1} \cup X_{2}$. (In general $W_{d}$ will be the result of attaching handles of dimension less than or equal to $d$ to $X_{1} \times I$ and $\partial W_{d}=X_{1} \cup X_{d}$.)

Since the attaching circles for the two handles are nullhomologous, $H_{2}\left(W_{2}, X_{1}\right)=H_{2}\left(W_{2}\right)$ is free abelian. Also $H_{2} W_{2}=H_{2} X_{2}$ since $W_{2}$ is obtained from $X_{2}$ by adding $(m-1)$-handles and $m-1>3$. Thus we can attach 3-handles to $W_{2}$ along $X_{2}$ to kill these generators (the Hopf sequence and $\mathrm{H}_{2}(\mathrm{Z})=0$ shows that $\mathrm{H}_{2} \mathrm{X}_{2}$ is all spherical). Again these handles can be attached along 2 -spheres in the complement of $f\left(\mathbf{S}^{p}\right)$ since $p \leq m-3$. See Figure 2.

(One word about framing the surgeries: since the manifold $X_{1}$ is framed, each surgery can be framed in such a way that the surgered manifolds are again framed. This is a fact due to Milnor [Mi2]. Thus it suffices to find an embedded spherical representative to kill any homotopy class below the middle dimension.)

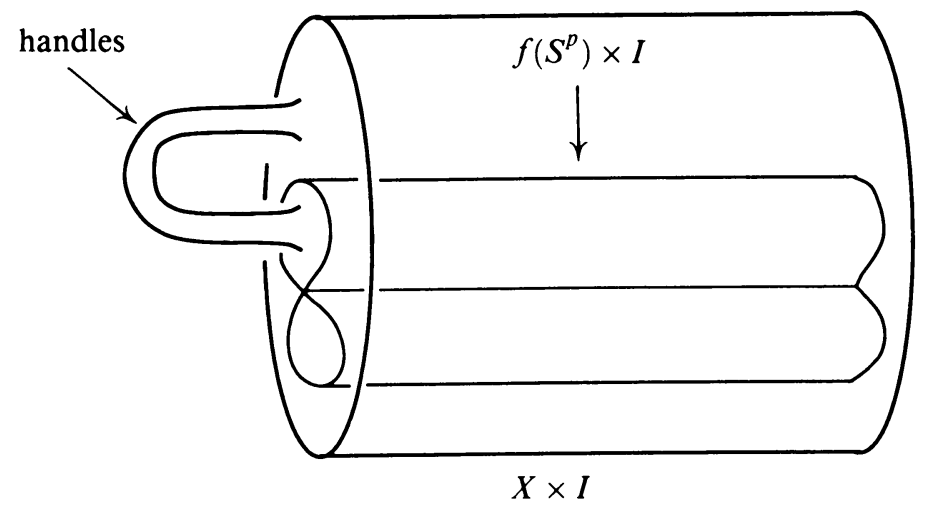

FIGURE 2

One easily sees that $H_{*}\left(W_{3}, X_{1}\right)=0$. Glueing $V \times \mathbf{I}$ to $W_{3}$ constructs a simply-connected $h$-cobordism because $\pi_{1} X_{1}$ is normally generated by a meridian and because

$$
H_{*}\left(W_{3} \cup(V \times \mathbf{I}), X_{1} \cup V\right) \cong H_{*}\left(W_{3}, X_{1}\right)=0 .
$$

Hence there exists a diffeomorphism of $W_{3} \cup(V \times \mathbf{I})$ with $\mathbf{S}^{m} \times \mathbf{I}$ which we may take to be the identity on $X_{1} \cup V=\mathbf{S}^{m}$. Furthermore, $X_{3}$ has the 1-homotopy type of $\mathbf{S}^{1}$.

Suppose by induction that we have attached handles of dimension less than or equal to $d$ to $\left(X_{1}-f\left(\mathbf{S}^{p}\right)\right) \times \mathbf{I}$ so that

1. $H_{*}\left(W_{d}, X_{1}\right)=0$.

2. $\mathbf{S}^{m} \times \mathbf{I} \cup$ (handles) is diffeomorphic to $\mathbf{S}^{m} \times \mathbf{I}$.

3. $\pi_{i}\left(W_{d}\right) \cong \pi_{i} \mathbf{S}^{1}$ for $i \leq d-2$. 
Notice that $W_{d}$ is obtained from $X_{d}$ by adding handles of dimension greater than or equal to $(m+1)-d$ whence $\pi_{i} X_{d}=\pi_{i} W_{d}$ and $H_{i} X_{d}=H_{i} W_{d}$ for $i \leq m-d-1$.

If at this point $d+p<m$, then we continue the construction as follows. First, $2 d<m$ so that $\pi_{d-1} W_{d}=\pi_{d-1} X_{d}$. By Alexander duality $H_{d-1}\left(X_{d}\right)=0$, so that one can attach $d$-handles to $X_{d}$ to kill $\pi_{d-1}\left(W_{d}\right)$. This produces some torsion free elements in the $d$ th homology, which we kill by attaching $(d+1)$ handles. Of course one must check that these elements are spherical. (This is not immediate. There is a small computation involved which relies on the fact that $m \geq 2 d$ and on the exact sequence

$$
\pi_{d} Y \rightarrow H_{d} Y \rightarrow H_{d}\left(\pi_{1} Y\right)
$$

valid for any space $Y$ with a $(d-1)$-connected universal cover. We omit it here to avoid introducing new notation.) Again this can be accomplished by attaching handles to the complement of $f\left(\mathbf{S}^{p}\right)$.

As before, $H_{*}\left(W_{d+1}, X_{1}\right)=0$ since homologically we attached $d$-cells along nullhomologous $(d-1)$-spheres, then killed the resulting $d$ homology. One concludes that $W_{d+2}$ is a homology cobordism and so $W_{d+2} \cup(V \times \mathbf{I})$ is an $h$-cobordism of $\mathbf{S}^{m}$ to itself. Finally, since handles of dimension greater than or equal to $m-d$ were added to $X_{d+1}$ to get $W_{d+1}$,

$$
\pi_{i} X_{d+1}=\pi_{i} W_{d+1} \quad \text { for } i \leq m-d-2
$$

and

$$
\pi_{i} W_{d+1}=\pi_{i} \mathbf{S}^{1} \quad \text { for } i \leq d-1 .
$$

We have now completed the induction step and so we can construct a concordance of $f\left(\mathbf{S}^{p}\right) \cup \mathbf{S}^{m-2}$ in $\mathbf{S}^{m}$ from the original link map $f$ to a link map $g: \mathbf{S}^{p} \cup \mathbf{S}^{m-2} \rightarrow \mathbf{S}^{m}$, say, so that $\pi_{i}\left(\mathbf{S}^{m}-g\left(\mathbf{S}^{m-2}\right)\right)=\pi_{i} \mathbf{S}^{1}$ for $i \leq m-p-2$.

We now wish to turn this concordance into a homotopy to finish the proof. We use the principle "concordance implies isotopy in codimension 3 or more". More precisely, call the concordance constructed above $W$ and notice that $W-(U \times \mathbf{I})$ is a simply connected $h$-cobordism with a product structure on the boundary. Thus we can find a product structure on all of $W-(U \times \mathbf{I})$ extending the one along $\partial U \times \mathbf{I}$. Use this product structure to project the concordance of $\mathbf{S}^{m-2}$ in $W$ into $\partial_{-} W$. This gives a homotopy of $\mathbf{S}^{m-2}$ in $\mathbf{S}^{m}-f\left(\mathbf{S}^{p}\right)$ from the original link map to $g$.

Lemma 5.3. If $f: \mathbf{S}^{p} \cup \mathbf{S}^{m-2} \rightarrow \mathbf{S}^{m}$ is a link map which embeds $\mathbf{S}^{m-2}$, then $\sigma(f)=0$,

We will postpone the proof of this fact until the end of this section.

Proof of Theorem 5.1. Let $\tilde{f}: \mathbf{S}^{p} \rightarrow \tilde{X}$ be a lift of $f: \mathbf{S}^{p} \rightarrow X=\mathbf{S}^{m}-\mathbf{S}^{m-2}$ to the $\mathbf{Z}$ cover, $\widetilde{X}$, of $X$. By Lemma 5.2 we may assume that $\tilde{X}$ is $(m-p-2)$ connected. The main theorem of Haefliger in [Hae] implies now that $\tilde{f}$ is 
homotopic to an embedding. By projecting the homotopy and lifting again, we may therefore change $f$ by a link homotopy so that $\underset{f}{\tilde{f}}$ is an embedding.

The problem now is to remove the intersections of $\widetilde{f}$ with its translates in $\tilde{X}$. Consider the intersection $D_{k}$ of $\tilde{f}\left(\mathbf{S}^{p}\right)$ with $t^{k} \cdot \tilde{f}\left(\mathbf{S}^{p}\right)$ for some $k>0$. In the specified range, the method for removing double points is to find a nullbordism $W_{k}$ of $D_{k}$, then to find a suitably framed embedding of $W_{k} \times \mathbf{I} /(d, t) \sim\left(d, t^{\prime}\right)$, $d \in D_{k}$, in $\tilde{X}$. One then slides $\tilde{f}\left(\mathbf{S}^{p}\right)$ along the I factor of $t^{k} \cdot \tilde{f}\left(\mathbf{S}^{p}\right)$. (The example to keep in mind is the Whitney trick, which corresponds to the case when the dimension of $D_{k}$ is zero.) This procedure can be completed if $\sigma_{k}$ is zero; indeed, $\sigma_{k}$ is exactly the obstruction to finding $W_{k}$ and framing $W_{k} \times \mathbf{I}$ appropriately. The details of this construction are found in Hatcher and Quinn's paper [HQ]. Their results can also be used directly in the following way: They define an obstruction in $\Omega_{2 p-m}(\Lambda \widetilde{X} ; \xi)(\Lambda \widetilde{X}$ denotes the loop space of $\tilde{X}$ and $\xi$ is a bundle which depends on the tangent bundles of $\tilde{X}$ and $\mathbf{S}^{p}$ ). They show this obstruction vanishes if and only if it is possible to regularly homotop $\tilde{f}\left(\mathbf{S}^{p}\right)$ off $t^{k} \cdot \tilde{f}\left(\mathbf{S}^{p}\right)$. But under the connectivity assumptions, the inclusion $\Omega_{2 p-m}^{f r} \rightarrow$ $\Omega_{2 p-m}(\Lambda \tilde{X} ; \xi)$ is an isomorphism which takes $\sigma_{k}$ to their obstruction. Hence if $\sigma_{k}$ is zero we may find a correctly framed $W_{k} \times \mathbf{I}$.

Thus we may find embeddings of $W_{k} \times \mathbf{I}$ for each $k$; by general position these may be chosen so that they are embedded and pairwise disjoint in $X$. The "generalized Whitney move" along the $W_{k} \times \mathbf{I}$ is a regular homotopy of $f\left(\mathbf{S}^{p}\right)$ to an immersion satisfying $\tilde{f}\left(\mathbf{S}^{p}\right) \cap t^{k} \cdot \tilde{f}\left(\mathbf{S}^{p}\right)=\varnothing$ for all $k$ so that $f: \mathbf{S}^{p} \rightarrow X$ is an embedding.

We have now found a link homotopy to an embedded link. We can now use the main result of [Ki2] which states that an embdded link $\mathbf{S}^{p} \cup \mathbf{S}^{m-2} \subset \mathbf{S}^{m}$ is nullhomotopic if $3 p+4 \leq 2 m$.

We need to prove Lemma 5.3. This will be done by introducing an "equivariant homotopy intersection pairing" on a knot complement $X$ or, more generally, on the complement of any codimension two cycle in $\mathbf{S}^{m}$ :

$$
\langle,\rangle: \pi_{p}(X) \times \pi_{q}(X) \rightarrow \pi_{p+q-m}^{s}\left[t, t^{-1}\right],
$$

which is a generalization of $\sigma$.

Lemma 2.3 implies that there is a well-defined function

$$
\pi_{p}(X) \rightarrow\left\{\text { regular homotopy classes of immersions of } \mathbf{S}^{p} \text { in } X\right\}
$$

taking a homotopy class $\beta$ to the unique regular homotopy class of an immersion $f: \mathbf{S}^{p} \rightarrow X$ such that $[f]=\beta$ and $i \circ f: \mathbf{S}^{p} \rightarrow X \subset \mathbf{S}^{m}$ is regularly homotopic to the standard inclusion.

Let $\widetilde{X}$ be the Z-cover of $X$ and let $f: \mathbf{S}^{p} \rightarrow X$ and $g: \mathbf{S}^{q} \rightarrow X$ be two transverse immersions representing elements of $\pi_{p} X$ and $\pi_{q} X$. Let $\tilde{f}$ and $\tilde{g}$ be the lifts of $f$ and $g$ to $\tilde{X}$. Let $t: \tilde{X} \rightarrow \tilde{X}$ denote the covering translation. 
Define the double point manifold

$$
D_{n}=\left\{(x, y) \in \mathbf{S}^{p} \times \mathbf{S}^{q} \mid \tilde{f}(x)=t^{n} \tilde{g}(y)\right\}=\left(\tilde{f} \times t^{n} \widetilde{g}\right)^{-1}\left(\Delta_{\tilde{X}}\right) .
$$

The canonical stable framings of the normal bundle of $\mathbf{S}^{p} \times \mathbf{S}^{q}$ and the tangent bundle of $\widetilde{X}$ give a framing $\mathscr{F}$ of the normal bundle of $D_{n}$, as described in $\S 3$. Thus we can define

$$
\tilde{f} \cdot t^{n} \widetilde{g}=\left[D_{n}, \mathscr{F}\right] \in \Omega_{p+q-m}^{f r}=\pi_{p+q-m}^{s} .
$$

Definition 5.4.

$$
\langle f, q\rangle=\sum_{n \in \mathbf{Z}}\left(\tilde{f} \cdot t^{n} \tilde{g}\right) t^{n} \in \pi_{p+q-m}^{s}\left[t, t^{-1}\right]
$$

Notice that $\pi_{p+q-m}^{s}\left[t, t^{-1}\right]$ is a $\mathbf{Z}\left[t, t^{-1}\right]$-module. Is has an involution $\alpha \mapsto$ $\bar{\alpha}$ defined by $\bar{t}=t^{-1}$.

The following properties of this pairing are easily checked:

1. $\left\langle f+f^{\prime}, g\right\rangle=\langle f, g\rangle+\left\langle f^{\prime}, g\right\rangle$.

2. $\langle\alpha f, g\rangle=\alpha\langle f, g\rangle$ for $\alpha \in \mathbf{Z}\left[t, t^{-1}\right]$.

3. $\langle g, f\rangle=(-1)^{p q} \frac{g\rangle, g\rangle}{\langle f}$.

It is obvious from the definition that if $f: \mathbf{S}^{p} \cup \mathbf{S}^{m-2} \rightarrow \mathbf{S}^{m}$ is a link map which embeds the codimension two component, then $\sigma(f)=0$ if $\langle f, f\rangle=0$.

Theorem 5.5. Let $f: \mathbf{S}^{p} \cup \mathbf{S}^{m-2} \rightarrow \mathbf{S}^{m}$ be a link map which embeds the codimension two components. If $3 p \leq 2 m-4$, then $\sigma(f)=0$.

Proof. By Lemma 5.2 we may assume that $\tilde{X}$ is $(m-p-2)$-connected. A theorem of Hirschhorn (Theorem 1 of $[\mathrm{H}])$ states that in this case $H_{k}\left(\mathbf{Z} ; \pi_{i} X\right)=0$ for all $k$ if $i \leq 2(m-p-2)$. This is equivalent to saying that the homomorphism $1-t: \pi_{i} X \rightarrow \pi_{i} X$ is an automorphism.

Thus, since $p \leq 2(m-p-2)$, there exist elements $f_{l} \in \pi_{p} X$ so that $f=$ $(1-t)^{l} f_{l}$ for each positive integer $l$. But then $\langle f, f\rangle=(1-t)^{l}\left\langle f_{l}, f\right\rangle$ for arbitrarily large $l$; this can happen only if $\langle f, f\rangle=0$. Thus $\sigma(f)=0$.

Since $\sigma=0$ whenever $\mathbf{S}^{m-2}$ is embedded, the double point manifold of $\mathbf{S}^{p}$ cannot "link" $\mathbf{S}^{m-2}$ in $\mathbf{S}^{m}$. If we take an immersion $\mathbf{S}^{p} \rightarrow \mathbf{S}^{m}$ given by Lemma 4.2 , then we see that a class in $\pi_{m-2}\left(\mathbf{S}^{m}-f\left(\mathbf{S}^{p}\right)\right)$ with nonzero image in $H_{m-2}\left(\mathbf{S}^{m}-f\left(\mathbf{S}^{p}\right)\right)$ cannot be represented by an embedding.

\section{6}

In this section we will consider the special case of link maps of two-spheres in $\mathbf{S}^{4}$. It is conjectured that Theorem 5.1 holds in this dimension also, that is, a link map $f: \mathbf{S}^{2} \cup \mathbf{S}^{2} \rightarrow \mathbf{S}^{4}$ which embeds one of the components is nullhomotopic. As usual, the higher-dimensional arguments which involve some version of the Whitney trick fail in this case. In fact, we will give an example below which shows that the Whitney trick cannot work in this setting. 


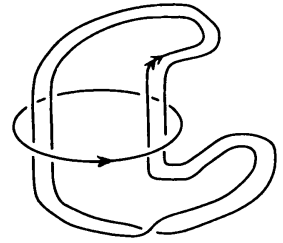

$t=0$

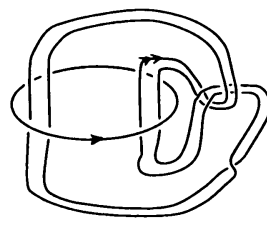

$t=\frac{1}{2}$

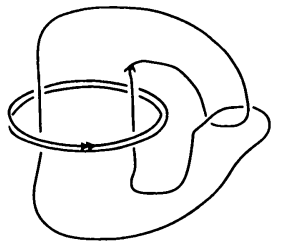

$t=\frac{3}{4}$

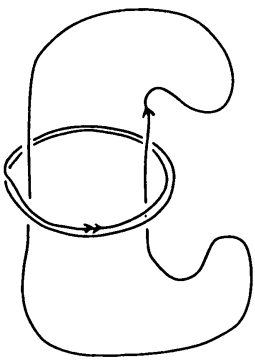

$t=1$

FiguRE 3

We remind the reader of some of the ideas of [Kil]. Write $f: \mathbf{S}_{+}^{2} \cup \mathbf{S}_{-}^{2} \rightarrow \mathbf{S}^{4}$ where the signs distinguish the components. By considering the double points of $f\left(\mathbf{S}_{+}^{2}\right)$ in $\mathbf{S}^{4}-f\left(\mathbf{S}_{-}^{2}\right)$ we define $\sigma_{+} \in \Omega_{0}^{f r}[t]=\mathbf{Z}[t]$. But one can also consider the double points of $f\left(\mathbf{S}_{-}^{2}\right)$ in the complement of $f\left(\mathbf{S}_{+}^{2}\right)$ to obtain another invariant $\sigma_{-}$. Thus

$$
\sigma=\sigma_{+} \oplus \sigma_{-}: L M_{2,2}^{4} \rightarrow \mathbf{Z}[t] \oplus \mathbf{Z}[t] .
$$

Since $\Omega_{0}^{f r}(*)=\Omega_{0}(*)=H_{0}(*)=\mathbf{Z}$, the framed bordism class of an isolated double point is just the sign of this double point. Thus one could just as well define $\sigma_{+}$by the formula

$$
\sigma_{+}=\sum_{x \in \text { double points of } f\left(\mathbf{S}_{+}^{2}\right)} \operatorname{sign}(x) \cdot\left(t^{\left|\alpha_{x}\right|}-1\right),
$$

where $\alpha_{x} \in \mathbf{Z}=H_{1}\left(\mathbf{S}^{4}-f\left(\mathbf{S}_{-}^{2}\right)\right)$ is the homology class of a loop in $f\left(\mathbf{S}_{+}^{2}\right)$ changing branches at $x$.

We will show that if $f$ embeds the first component then both $\sigma_{+}$and $\sigma_{-}$ vanish. Thus Theorem 5.5 holds for link maps in this dimension.

This has the following interesting consequence. Suppose $f: \mathbf{S}_{+}^{2} \cup \mathbf{S}_{-}^{2} \rightarrow \mathbf{S}^{4}$ is a link map for which $\sigma=(0, p(t))$ with $p(t)$ nonzero. There are many such examples; we give one construction which the reader can easily generalize. Figure 3 shows an example of a link map, $g$, with $\sigma(g)=\left(4-4 t, t^{2}-1\right)$. We use the "moving picture" method to represent the spheres in $\mathbf{R}^{4} \subset \mathbf{S}^{4}$. Let $h$ be the link map of Figure 1 with $\sigma(h)=(t-1,1-t)$. Then the link map $f=g \# h \# h \# h \# h$ has $\sigma(f)=\left(0, t^{2}-4 t+3\right)$.

Given such a link map, we may homotop $\mathbf{S}_{-}^{2}$ in the complement of $\mathbf{S}_{+}^{2}$ using finger moves (the reverse of the Whitney move) to abelianize the fundamental group of $\mathbf{S}^{4}-f\left(\mathbf{S}_{-}^{2}\right)$, so that $\pi_{1}\left(\mathbf{S}^{4}-f\left(\mathbf{S}_{-}^{2}\right)\right)=\mathbf{Z}$ [Kil]. Furthermore, we may perturb $f\left(\mathbf{S}_{+}^{2}\right)$ so that $\sum \operatorname{sign}(x)=0$ by a small (local) homotopy. But then 
$\sigma_{+}(t)+\sigma_{+}\left(t^{-1}\right)$ is the usual obstruction in $\mathbf{Z}[\mathbf{Z}]=\mathbf{Z}\left[\pi_{1}\left(\mathbf{S}^{4}-f\left(\mathbf{S}_{-}^{2}\right)\right)\right]$ to homotop $\mathbf{S}_{+}^{2}$ to an embedding in $\mathbf{S}^{4}-f\left(\mathbf{S}_{-}^{2}\right)$. If the vanishing of this obstruction sufficed to homotop $\mathbf{S}_{+}^{2}$ to an embedding (as it does in higher dimensions) then the resulting link map would have one component embedded but $\sigma$ nonzero. Thus one cannot find a homotopy of $\mathbf{S}_{+}^{2}$ to an embedding even though the usual group ring obstruction vanishes.

We now show that Theorem 5.5 holds in this dimension also.

Theorem 6.1. Let $f: \mathbf{S}_{+}^{2} \cup \mathbf{S}_{-}^{2} \rightarrow \mathbf{S}^{4}$ be a link map which embeds $\mathbf{S}_{+}^{2}$. Then $\sigma_{-}=0$.

Proof. The proof is similar to the proof of Theorem 5.5. Let $X_{+}$be the complement of $f\left(\mathbf{S}_{+}^{2}\right)$ and let $\widetilde{X}_{+}$be its infinite cyclic cover. As before, $\sigma_{-}(f)$ is determined by $\langle f, f\rangle$, where

$$
\langle,\rangle: \pi_{2}\left(\tilde{X}_{+}\right) \times \pi_{2}\left(\tilde{X}_{+}\right) \rightarrow \mathbf{Z}\left[t, t^{-1}\right]
$$

is the homotopy intersection form. However, since the sign of a self-intersection point determines its framed bordism class, this intersection form is defined not just on $\pi_{2}\left(\tilde{X}_{+}\right)$but on $H_{2}\left(\tilde{X}_{+}\right)$. The homology groups of the infinite cyclic cover of a knot are torsion over the ring $\mathrm{Z}\left[t, t^{-1}\right][\mathrm{L}]$ so that as before we may conclude that $\langle$,$\rangle is the zero pairing. Thus \sigma_{-}=0$ as claimed.

There are many interesting questions which remain unanswered. We mention a few.

Notice that $\sigma$ can be defined for multiple component link maps by considering the $\mathbf{Z}^{r}$ covers where $r$ is the number of codimension two components. However, no interesting examples are know. Notice that if $f: S_{1}^{2} \cup \cdots \cup S_{n}^{2} \rightarrow \mathbf{S}^{4}$ is a link map, then $\sigma$ lies in $\mathbf{Z}\left[t_{1}, \ldots, t_{n-1}\right]$.

Question. Are there link maps $f: \mathbf{S}^{2} \cup \mathbf{S}^{2} \cup \mathbf{S}^{2} \rightarrow \mathbf{S}^{4}$ so that one of the three $\sigma$ invariants has mixed terms?

The invariant $\sigma$ for $f: \mathbf{S}_{+}^{2} \cup \mathbf{S}_{-}^{2} \rightarrow \mathbf{S}^{4}$ is related to certain obstructions for embedded links $\mathbf{S}^{1} \cup \mathbf{S}^{1} \subset \mathbf{S}^{3}$ to be concordant to boundary links [J]. Since it is conjectured that higher dimensional links are concordant to boundary links, one may ask:

Question. Is $\sigma=0$ for all $f: \mathbf{S}^{m-2} \cup \mathbf{S}^{m-2} \rightarrow \mathbf{S}^{m}$ with $m>4$ ? Are all such links nullhomotopic? Are embedded links in pure codimension two nullhomotopic?

Boundary links $S^{2} \cup S^{2} \subset \mathbf{S}^{4}$ are nullhomotopic [Kil].

Question. Are boundary links nullhomotopic in all dimensions?

The answer to this question is no for boundary link maps, i.e. link maps which bound disjoint mapped in Seifert surfaces, at least for link maps

$$
f: \mathbf{S}^{m-2} \cup \mathbf{S}^{m-2} \rightarrow \mathbf{S}^{m} \text { with } m>4 .
$$


The counterexamples are link maps whose Seifert surfaces are punctured $\mathbf{C} \mathbf{P}^{(m-1) / 2}$. The answer to the following question may still be yes.

Question. Are boundary link maps which bound nullbordant mapped in Seifert surfaces nullhomotopic? Notice that this would answer the previous question.

I wish to thank J. Levine and D. Rolfsen for suggesting some of these questions.

\section{REFERENCES}

[FR] R. Fenn and D. Rolfsen, Spheres may link homotopically in 4-sphere, J. London Math. Soc. (2) 34 (1986), 177-184.

[Hab] N. Habegger, On linking coefficients, Proc. Amer. Math. Soc. 96 (1986), 353-359.

[J] G. T. Jin, Invariants of two component links, Thesis, Brandeis University, 1988.

[Hae] A. Haefliger, Plongements differentiables de variétés dans varités, Comment. Math. Helv. 36 (1961), 47-82.

[HQ] A. Hatcher and F. Quinn, Bordism invariants of intersectins of submanifolds, Trans. Amer. Math. Soc. 200 (1974), 327-344.

[Hi] M. Hirsch, Immersions of manifolds, Trans. Amer. Math. Soc. 93 (1959), 242-276.

[H] P. Hirschhorn, The stable homotopy type of knot complements, Illinois J. Math. 23 (1979), 128-133.

[Hu] J. Hughes, Thesis, University of California, Berkeley, 1983.

[Ki] P. Kirk, Link maps in the 4-sphere, Proceedings of the Second Siegen Topology Symposium 1987, Lecture Notes in Math., vol. 1350, Springer-Verlag, Berlin, Heidelberg and New York, 1988, pp. 44-86.

[Ki2] _ Embedded links with one codimension two component are nullhomotopic in the metastable range, J. London Math. Soc. (to appear).

[Kol] U. Koschorke, Higher order invariants for higher dimensional link maps, Lecture Notes in Math., vol. 1172, Springer-Verlag, Berlin, Heidelberg and New York, 1984, pp. 116-128.

[Ko2] _ On link maps and the geometry of their invariants, Manuscripta Math. 61 (1988), 383-415.

[Ko3] _ Multiple point invariants of link maps, Proceedings of the Second Siegen Topology Symposium 1987, Lecture Notes in Math., vol. 1350, Springer-Verlag, Berlin, Heidelberg and New York, 1988, pp. 44-86.

[Ko4] _ On link maps and their homotopy classification, preprint, 1988.

[KoR] U. Koschorke and D. Rolfsen, Higher dimensional link operations and stable homotopy, preprint, 1987.

[KoS] U. Koschorke and B. Sanderson, Geometric interpretations of the generalized Hopf invariant, Math. Scand. 41 (1977), 199-217.

[L] J. Levine, Knot modules. I, Trans. Amer. Math. Soc. 229 (1977), 1-50.

[MR] W. S. Massey and D. Rolfsen, Homotopy classification of higher dimensional links, Indiana Univ. Math. J. 34 (1986), 375-391.

[Mi1] J. Milnor, Link groups, Ann. of Math. (2) 59 (1954), 177-195.

[Mi2] J. Milnor, A procedure for killing homotopy groups of differentiable manifolds, Proc. Sympos. Pure Math., vol. 3, Amer. Math. Soc., Providence, R.I., 1961, pp. 39-55. 
[Sc] G. P. Scott, Homotopy links, Abh. Math. Sem. Hamburg 32 (1968), 186-190.

[W] H. Whitney, The self-intersections of a smooth n-manifold in $2 n$ space, Ann. of Math. (2) 45 (1944), 220-246.

Department of Mathematics, Brandeis University, Waltham, Massachusetts 02254

Current address: Department of Mathematics, California Institute of Technology, Pasadena, California 91125 